\title{
Ordovician-Silurian rift-passive margin on the Mexican margin of the Rheic Ocean overlain by Carboniferous-Permian periarc rocks: Evidence from the eastern Acatlán Complex, southern Mexico
}

\author{
Miguel Morales-Gámez ${ }^{\text {a }}$, J. Duncan Keppie ${ }^{\mathrm{a}, *}$, Marc Norman ${ }^{\mathrm{b}}$ \\ a Departamento de Geología Regional, Instituto de Geología, Universidad Nacional Autónoma de México, C.P. 04510 México D.F., Mexico \\ ${ }^{\mathrm{b}}$ Research School of Earth Sciences, Australian National University, Mills Road, Building 61, Canberra, ACT 0200, Australia
}

Received 16 May 2007; received in revised form 22 October 2007; accepted 25 January 2008

Available online 8 February 2008

\begin{abstract}
In the Xayacatlán area of the Acatlán Complex in southern Mexico, several, N-S, fault-bounded packages of rocks occur (from west to east): (i) greenschist facies, interbedded psammites and pelites (Huerta Unit): the youngest concordant detrital zircon in the western part of the unit is $455 \pm 4 \mathrm{Ma}$; (ii) greenschist-amphibolite facies arkoses and psammites (Amate Unit): the youngest concordant detrital zircon gave a ${ }^{206} \mathrm{~Pb} /{ }^{238} \mathrm{U}$ age of $902 \pm 14 \mathrm{Ma}$, and older concordant ages of $\sim 900-1300 \mathrm{Ma}$; (iii) low grade clastic rocks and marbles (Permian Tecomate Formation); and (iv) greenschist facies psammites, pelites and thin tholeiitic mafic slices (Salada Unit): the youngest concordant detrital zircon yielded a ${ }^{206} \mathrm{~Pb} /{ }^{238} \mathrm{U}$ age of $352 \pm 3 \mathrm{Ma}$ and older concordant ages of $\sim 434-485 \mathrm{Ma}, 511-630 \mathrm{Ma}$, and 920-1200 Ma. The eastern part of the Huerta Unit is cut by pegmatite that yielded an almost concordant ${ }^{206} \mathrm{~Pb} /{ }^{238} \mathrm{U}$ zircon age of $464 \pm 4 \mathrm{Ma}$ (a mean age of the four almost concordant young zircons is $\sim 485.5 \mathrm{Ma}$ ) interpreted as the time of intrusion, and inherited zircons with concordant ages of $\sim 530-580$ Ma and $\sim 910-1200 \mathrm{Ma}$. These data suggest that the Huerta Unit ranges in age from Early (possibly older) to Late Ordovician. The Amate Unit is cut by granitic dikes, in which the youngest concordant ${ }^{206} \mathrm{~Pb} /{ }^{238} \mathrm{U}$ zircon ages are $452 \pm 6 \mathrm{Ma}$ and $447 \pm 3 \mathrm{Ma}$ interpreted as the time of intrusion, and older inherited zircon ages with concordant ${ }^{206} \mathrm{~Pb} /{ }^{238} \mathrm{U}$ ages in the range of $950-1250 \mathrm{Ma}$. The intrusive ages are similar to the $442 \pm 1 \mathrm{Ma}$ concordant $\mathrm{U}-\mathrm{Pb}$ age for mafic dikes that also cut the Amate Unit. These data constrain the depositional age of the Amate Unit between $~ 900$ Ma and latest Ordovician. The age of the Salada Unit is probably Carboniferous because it is structurally overlain by the Lower-Middle Permian Tecomate Unit. The preponderance of 510-630 Ma and 900-1250 Ma ages in both the detrital and inherited ages in some of the dated samples suggests a source in the adjacent and subjacent Maya and Oaxacan Complex, respectively, although a xenocrystic host rock source cannot be ruled out for the intrusive rocks. The igneous intrusions have been interpreted as part of a 480-440 Ma bimodal rift-related suite. Their synchroneity with deposition of the Huerta Unit (and possibly also the Amate Unit) suggests a rift-passive margin environment of deposition on the southern margin of the Rheic Ocean. On the other hand, the Salada Unit and the Tecomate Formation appear to be synchronous with a Permo-Triassic magmatic arc that developed on the western margin of Pangea.
\end{abstract}

(C) 2008 Elsevier B.V. All rights reserved.

Keywords: Acatlán Complex; Mexico; Ordovician; Permo-Carboniferous; Geochronology

\section{Introduction}

The Xayacatlán area is located in the eastern part of the Acatlán Complex (Fig. 1) (designated the Mixteca terrane by

\footnotetext{
* Corresponding author. Fax: +525556224290 .

E-mail addresses: morzuz@yahoo.com (M. Morales-Gámez), duncan@servidor.unam.mx (J.D. Keppie).
}

Campa and Coney, 1983). The Acatlán Complex has been interpreted to be a vestige of a Paleozoic ocean, however, which ocean is presently a matter of debate. Ortega-Gutiérrez et al. (1999) suggested that it was part of the Cambro-Ordovician Iapetus Ocean, whereas Keppie and Ramos (1999) proposed that it was part of the Ordovician-Devonian Rheic Ocean. Subsequently, Talavera-Mendoza et al. (2005) and VegaGranillo (2007) published a model that placed parts of the 


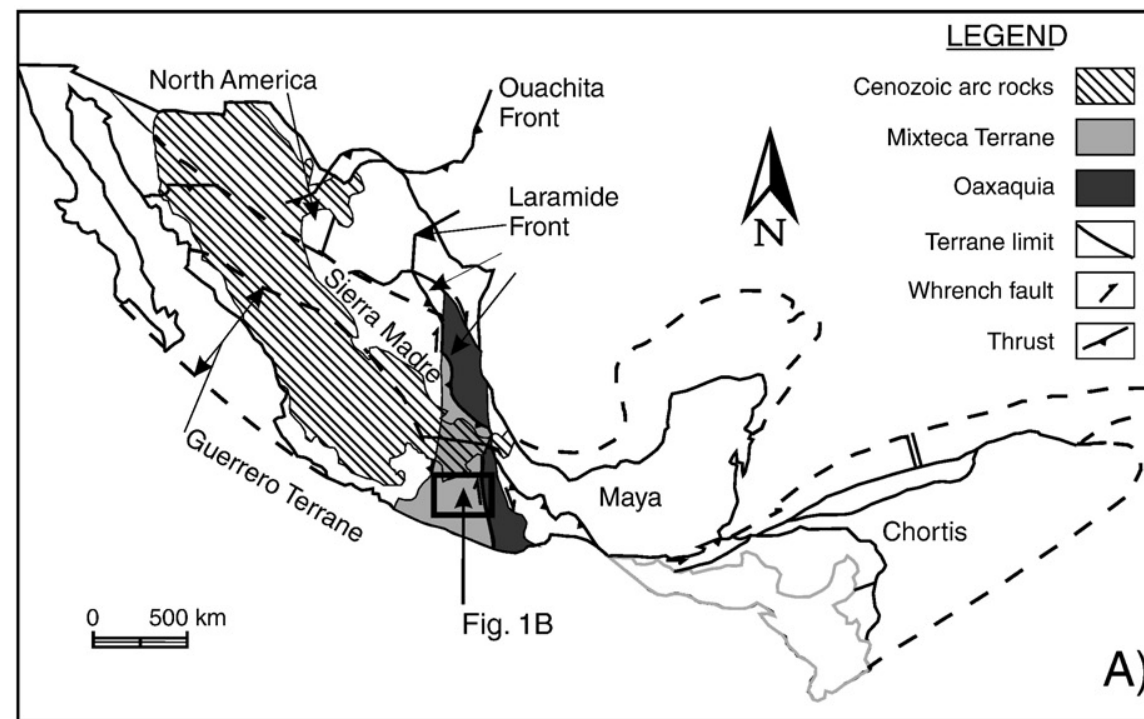

LEGEND FOR FIGURE 1B

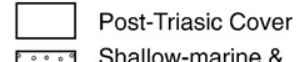

Continental strata

Acatlán Complex

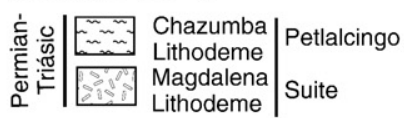

(2)

ஸे

空

के

Ordovician-Mississipian

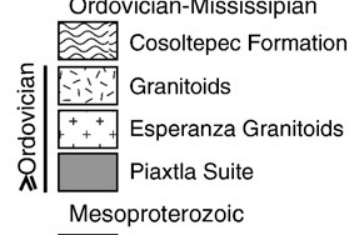

A)

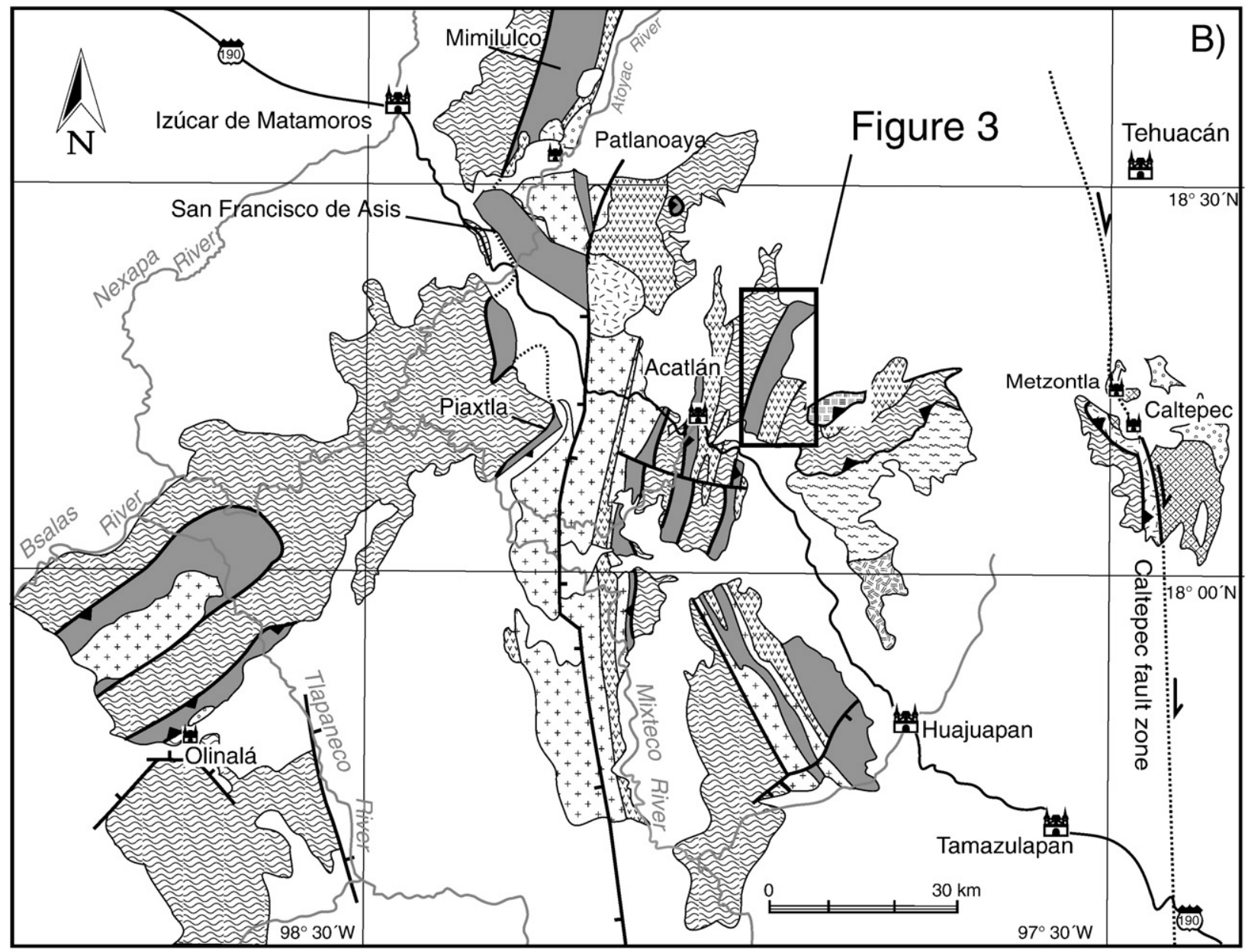

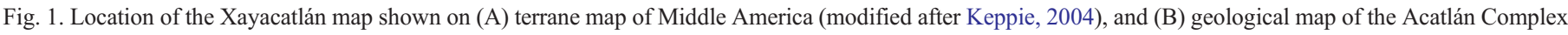
(modified after Keppie et al., 2006).

Acatlán Complex in three oceans: the El Rodeo unit and Lower Ordovician megacrystic granitoids were placed in the Bay Verte seaway between eastern Laurentia and an arc, the Xayacatlán Formation was attributed to the Iapetus Ocean, and the
Cosoltepec Formation was envisaged as a passive margin bordering Amazonia. A recent synthesis by Keppie et al. (2008a) proposes that the Acatlán Complex originated on the southern margin of the Rheic Ocean traveling with Amazonia to 


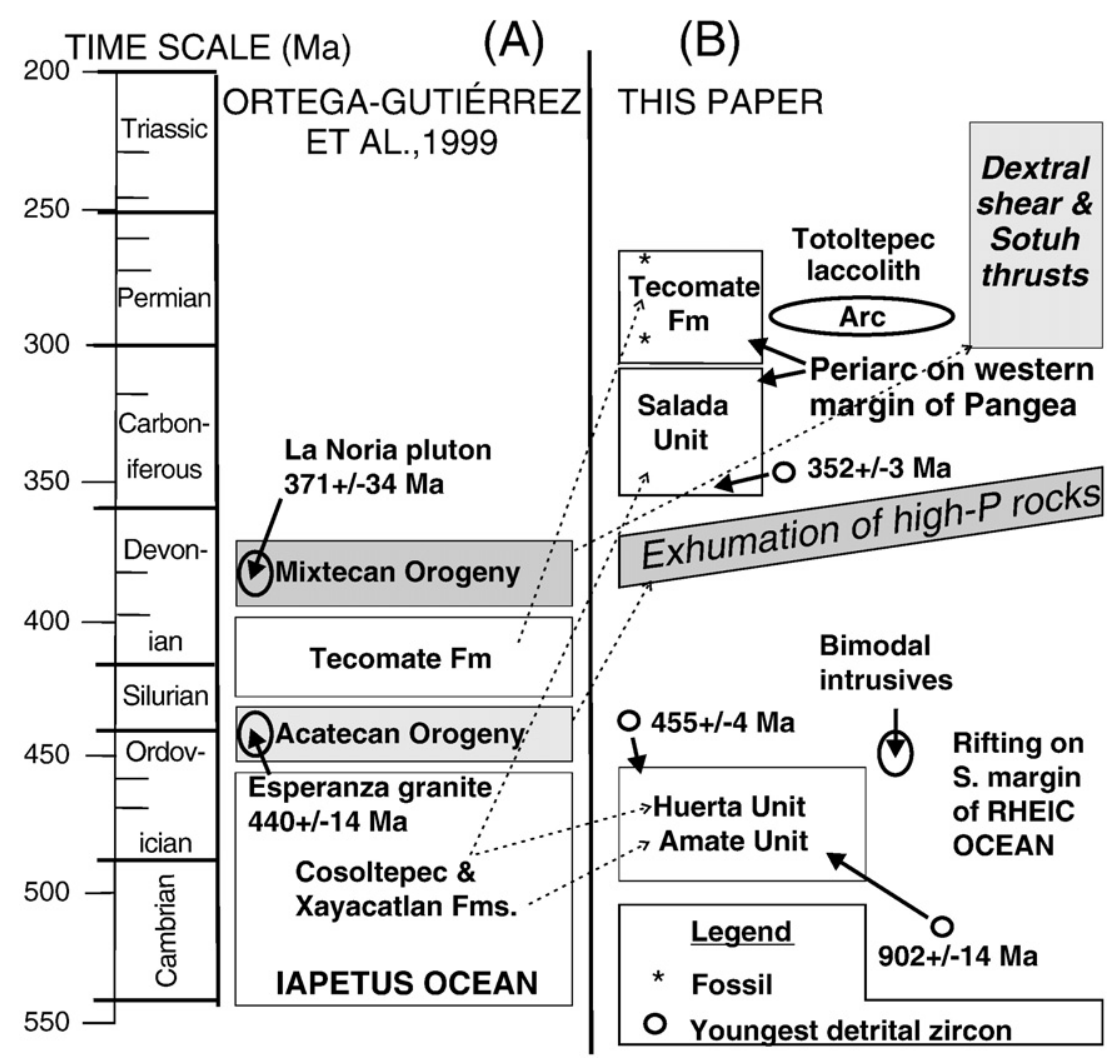

Fig. 2. Time-and-space diagram showing geological record of the Xayacatlán area: (A) after Ortega-Gutiérrez et al. (1999), and (B) this work.

end up on the active margin of the paleo-Pacific Ocean (Keppie et al., 2008a).

The Xayacatlán area is critical to resolution of this debate because it is the type area of the Xayacatlán Formation (OrtegaGutiérrez, 1975). Ortega-Gutiérrez (1975) described the Xayacatlán Formation as an assemblage of metapsammites, metapelites, metagabbros, amphibolites, and serpentinite associated with megacrystic granitoids (Esperanza granitoids) that had undergone eclogite facies metamorphism (Fig. 2A). This high pressure metamorphism was believed to be late Ordovician based on the inference that the megacrystic granitoids were decompression melts that yielded a lower intercept $\mathrm{U}-\mathrm{Pb}$ age of $440 \pm 14 \mathrm{Ma}$ (Ortega-Gutiérrez et al., 1999). During this tectonothermal event, the Xayacatlán Formation was believed to have been thrust over the low grade Petlalcingo Group consisting of the Magdalena Migmatite at the base, the Chazumba Formation, and the Cosoltepec Formation at the top. Following exhumation, these rocks were unconformably overlain by the Tecomate Formation (of supposedly Siluro-Devonian age), which was intruded by arcrelated rocks of Devonian age (based on a lower intercept age of $371 \pm 34 \mathrm{Ma}$ (poorly defined lower intercept U-Pb TIMS zircon age: Yañez et al., 1991) during a Middle-Upper Devonian orogeny called the Mixtecan Orogeny (Sánchez-Zavala et al., 2000). This was followed by deposition of Carboniferous and Permian rocks unconformably on the older rocks.

However, the eclogite facies metamorphism has since been dated directly as Mississippian in the northern part of the Acatlán Complex (Middleton et al., 2007; Elías-Herrera et al.,
2007), whereas the megacrystic granitoids are almost exclusively of Ordovician age (Sánchez-Zavala et al., 2004; TalaveraMendoza et al., 2005; Miller et al., 2007). Furthermore, the fossils and dated granitic pebbles in Tecomate Formation show it to be Early-Middle Permian, possibly extending into the Carboniferous in undated underlying rocks (Keppie et al., 2004a). The Magdalena and Chazumba units contain detrital zircons as young as Permian and Triassic, whereas the youngest detrital zircons in units included in the Cosoltepec Formation of the eastern Acatlán Complex are either Ordovician ( $455 \mathrm{Ma}$ : Keppie et al., 2004b, 2006) or Devono-Carboniferous ( 410 and/or 374 Ma: Talavera-Mendoza et al., 2005). This has led to a revision of the geological history of the Acatlán Complex that has been summarized by Nance et al. $(2006,2007)$ and Keppie et al. (2008a) involving:

(i) deposition of ?Cambro-Ordovician rift-passive margin sediments associated with intrusion of bimodal, riftrelated igneous rocks (Miller et al., 2007; Keppie et al., 2008b; Ramos-Arias et al., 2008);

(ii) deposition of latest Devonian-Permian-Triassic continental-shallow marine rocks (Vachard et al., 2000) that was synchronous with Mississippian exhumation of the high pressure rocks (Middleton et al., 2007), extensional and dextral shear on listric normal zones and $\mathrm{N}-\mathrm{S}$ vertical zones (Elías-Herrera and Ortega-Gutiérrez, 2002; RamosArias et al., 2008), and Permo-Triassic arc magmatism (Torres et al., 1999; Malone et al., 2002). 

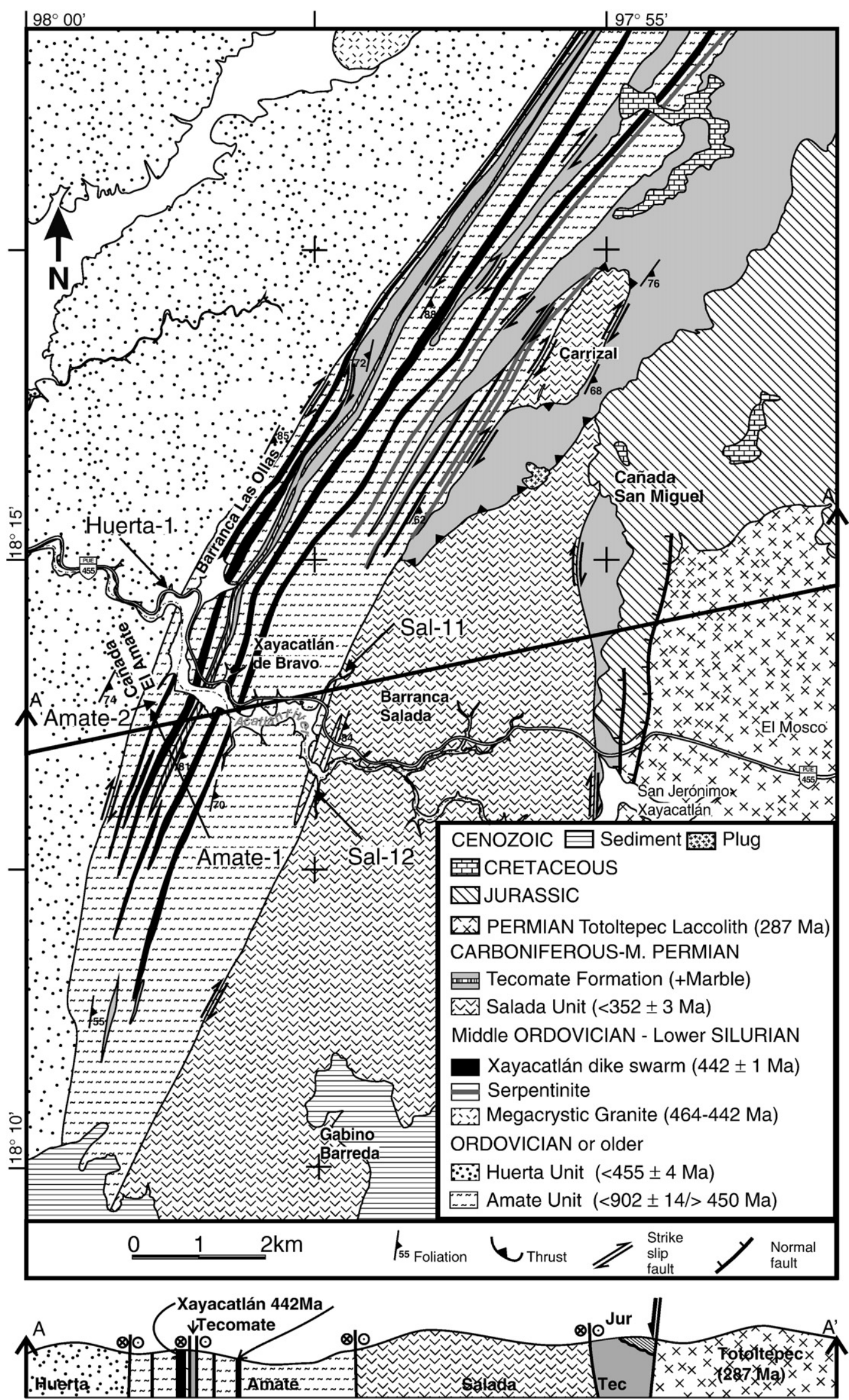

Fig. 3. Geological map of the Xayacatlán area. 
Table 1

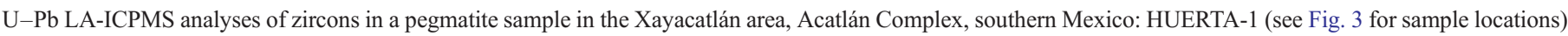

\begin{tabular}{|c|c|c|c|c|c|c|c|c|c|c|c|c|}
\hline Sample/analysis & ${ }^{206} \mathrm{~Pb} /{ }^{238} \mathrm{U}$ & $\begin{array}{l}\text { Percentage } \\
\text { error }\end{array}$ & ${ }^{207} \mathrm{~Pb} /{ }^{235} \mathrm{U}$ & $\begin{array}{l}\text { Percentage } \\
\text { error }\end{array}$ & ${ }^{207} \mathrm{~Pb} /{ }^{206} \mathrm{~Pb}$ & $\begin{array}{l}\text { Percentage } \\
\text { error }\end{array}$ & $\begin{array}{l}\text { Age, Ma } \\
{ }^{206} \mathrm{~Pb}^{238} \mathrm{U}\end{array}$ & $(2 \sigma)$ & ${ }^{207} \mathrm{~Pb} /{ }^{235} \mathrm{U}$ & $(2 \sigma)$ & ${ }^{207} \mathrm{~Pb} /{ }^{206} \mathrm{~Pb}$ & $(2 \sigma)$ \\
\hline \multicolumn{13}{|l|}{ HUERTA-1 } \\
\hline ap05e10rim & 0.1079 & 0.00086 & 0.9355 & 0.0092 & 0.06287 & 0.00062 & 659 & 10 & 671 & 10 & 702 & 42 \\
\hline ap05e11core & 0.1513 & 0.00123 & 1.4791 & 0.0213 & 0.07091 & 0.00078 & 908 & 14 & 922 & 17 & 954 & 44 \\
\hline ap05e12 & 0.197 & 0.0014 & 2.297 & 0.0372 & 0.08458 & 0.00132 & 1150 & 19 & 1211 & 23 & 1304 & 60 \\
\hline ap $05 \mathrm{e} 13$ & 0.0912 & 0.0007 & 0.7578 & 0.0213 & 0.06028 & 0.00189 & 565 & 14 & 573 & 25 & 612 & 134 \\
\hline ap05e14 & 0.117 & 0.0007 & 1.0857 & 0.0125 & 0.06732 & 0.00077 & 708 & 9 & 746 & 12 & 846 & 48 \\
\hline ap $05 \mathrm{e} 15$ & 0.0913 & 0.00041 & 0.7574 & 0.0081 & 0.06019 & 0.00062 & 563 & 5 & 573 & 9 & 610 & 44 \\
\hline ap $05 \mathrm{e} 16$ & 0.1127 & 0.00034 & 0.9879 & 0.0056 & 0.0636 & 0.00035 & 687 & 4 & 698 & 6 & 728 & 22 \\
\hline ap05f05 & 0.4287 & 0.0021 & 9.4091 & 0.0452 & 0.1592 & 0.00089 & 2299 & 20 & 2379 & 9 & 2446 & 20 \\
\hline ap05f06dark rim. & 0.0938 & 0.0004 & 0.7637 & 0.0082 & 0.05907 & 0.00066 & 578 & 5 & 576 & 9 & 568 & 48 \\
\hline ap05f07mottled.core. & 0.1249 & 0.00055 & 1.1849 & 0.0111 & 0.06883 & 0.00062 & 757 & 7 & 794 & 10 & 892 & 38 \\
\hline ap05f08 & 0.0855 & 0.00037 & 0.686 & 0.0062 & 0.05819 & 0.00056 & 529 & 5 & 530 & 7 & 536 & 40 \\
\hline ap05f09 & 0.0905 & 0.0009 & 0.7327 & 0.0155 & 0.05873 & 0.00143 & 563 & 16 & 558 & 18 & 556 & 106 \\
\hline ap05f10 & 0.3966 & 0.00238 & 8.5912 & 0.0481 & 0.15713 & 0.00074 & 2160 & 25 & 2296 & 10 & 2424 & 16 \\
\hline ap05f11 & 0.1165 & 0.00076 & 1.0801 & 0.0095 & 0.06726 & 0.00057 & 710 & 9 & 744 & 9 & 844 & 34 \\
\hline ap05f12 & 0.1746 & 0.00066 & 1.8474 & 0.0176 & 0.07673 & 0.00071 & 1036 & 8 & 1063 & 12 & 1114 & 38 \\
\hline ap05f13 & 0.1006 & 0.00056 & 0.8499 & 0.0097 & 0.0613 & 0.00067 & 617 & 7 & 625 & 11 & 648 & 48 \\
\hline ap05f14 & 0.0897 & 0.00045 & 0.7378 & 0.0081 & 0.05963 & 0.00069 & 554 & 6 & 561 & 9 & 590 & 50 \\
\hline ap05f15 & 0.0778 & 0.00036 & 0.6137 & 0.0071 & 0.05724 & 0.00065 & 483 & 5 & 486 & 9 & 500 & 50 \\
\hline ap05f16 & 0.4805 & 0.00221 & 12.3109 & 0.0665 & 0.18584 & 0.00095 & 2531 & 21 & 2628 & 10 & 2704 & 18 \\
\hline ap05g05 & 0.1732 & 0.00132 & 1.8143 & 0.0318 & 0.07599 & 0.00131 & 1030 & 16 & 1051 & 23 & 1094 & 70 \\
\hline ap05g06 & 0.092 & 0.00036 & 0.8328 & 0.0082 & 0.06569 & 0.00064 & 563 & 5 & 615 & 9 & 796 & 42 \\
\hline ap05g07 & 0.1685 & 0.00113 & 1.9705 & 0.0211 & 0.08484 & 0.00088 & 997 & 13 & 1105 & 14 & 1310 & 40 \\
\hline ap05g08 & 0.0933 & 0.00081 & 0.777 & 0.0184 & 0.06041 & 0.00154 & 575 & 10 & 584 & 21 & 618 & 110 \\
\hline ap05g09 & 0.1516 & 0.00091 & 1.4613 & 0.0178 & 0.06991 & 0.00073 & 911 & 11 & 915 & 15 & 924 & 44 \\
\hline ap05g10d.rim & 0.08 & 0.00035 & 0.6243 & 0.0066 & 0.05662 & 0.00061 & 496 & 4 & 493 & 8 & 476 & 48 \\
\hline ap05g111.core & 0.0804 & 0.00042 & 0.6502 & 0.0075 & 0.05864 & 0.00071 & 499 & 5 & 509 & 9 & 552 & 52 \\
\hline ap05g12 & 0.1042 & 0.00048 & 0.9385 & 0.0135 & 0.06536 & 0.00093 & 636 & 6 & 672 & 14 & 784 & 60 \\
\hline ap05g13 & 0.1057 & 0.00035 & 0.9022 & 0.0076 & 0.06193 & 0.00053 & 648 & 4 & 653 & 8 & 670 & 36 \\
\hline ap05g14 & 0.0937 & 0.00046 & 0.7813 & 0.0111 & 0.06048 & 0.00084 & 576 & 5 & 586 & 13 & 620 & 60 \\
\hline ap05g15 & 0.0904 & 0.00098 & 0.8729 & 0.0357 & 0.07007 & 0.00294 & 551 & 13 & 637 & 39 & 930 & 174 \\
\hline ap05g16 & 0.3259 & 0.00147 & 4.9909 & 0.0359 & 0.11108 & 0.00083 & 1820 & 18 & 1818 & 12 & 1816 & 28 \\
\hline ap05h05 & 0.2037 & 0.00065 & 2.2638 & 0.0152 & 0.08062 & 0.00052 & 1197 & 8 & 1201 & 9 & 1212 & 26 \\
\hline ap05h06 & 0.0851 & 0.00083 & 0.6911 & 0.0254 & 0.05889 & 0.00225 & 529 & 11 & 533 & 31 & 562 & 168 \\
\hline ap05h07 & 0.1748 & 0.00072 & 1.9479 & 0.0265 & 0.08084 & 0.00104 & 1030 & 9 & 1098 & 18 & 1216 & 52 \\
\hline ap05h08 & 0.205 & 0.00133 & 3.628 & 0.0395 & 0.12836 & 0.001 & 1205 & 15 & 1556 & 17 & 2074 & 28 \\
\hline ap05h09 & 0.18 & 0.00074 & 1.8704 & 0.0172 & 0.07538 & 0.00077 & 1069 & 9 & 1071 & 12 & 1078 & 40 \\
\hline ap05h10 & 0.1713 & 0.00081 & 1.7546 & 0.0223 & 0.07428 & 0.00091 & 1021 & 10 & 1029 & 16 & 1048 & 48 \\
\hline ap05h11 & 0.0746 & 0.00031 & 0.5913 & 0.0052 & 0.05748 & 0.00051 & 464 & 4 & 472 & 7 & 510 & 38 \\
\hline ap05h12 & 0.1381 & 0.00062 & 1.3176 & 0.009 & 0.06923 & 0.00046 & 832 & 8 & 853 & 8 & 904 & 28 \\
\hline ap05h13 & 0.0962 & 0.00047 & 0.787 & 0.0127 & 0.05935 & 0.00095 & 591 & 6 & 589 & 14 & 580 & 70 \\
\hline ap05h14 & 0.1546 & 0.00056 & 1.5503 & 0.0116 & 0.07273 & 0.00051 & 920 & 7 & 951 & 9 & 1006 & 28 \\
\hline ap05h15 & 0.095 & 0.00065 & 0.7901 & 0.0145 & 0.06036 & 0.00113 & 583 & 11 & 591 & 16 & 616 & 82 \\
\hline ap05h16 & 0.1179 & 0.00101 & 1.0652 & 0.024 & 0.06556 & 0.00146 & 716 & 12 & 736 & 24 & 792 & 94 \\
\hline ap05i05 & 0.4544 & 0.00204 & 11.5921 & 0.0545 & 0.18502 & 0.00104 & 2396 & 21 & 2572 & 9 & 2698 & 18 \\
\hline ap05i06 & 0.2908 & 0.00125 & 4.1756 & 0.0263 & 0.10415 & 0.00055 & 1645 & 14 & 1669 & 10 & 1698 & 20 \\
\hline ap05i07 & 0.2028 & 0.0014 & 2.2418 & 0.0365 & 0.08017 & 0.00135 & 1189 & 18 & 1194 & 23 & 1200 & 68 \\
\hline ap05i08 & 0.0686 & 0.00045 & 0.569 & 0.0075 & 0.06017 & 0.00079 & 427 & 6 & 457 & 10 & 608 & 56 \\
\hline ap05i09 & 0.1699 & 0.00093 & 1.7583 & 0.0222 & 0.07508 & 0.00104 & 1009 & 12 & 1030 & 16 & 1070 & 56 \\
\hline ap05i10 & 0.5104 & 0.00327 & 16.3265 & 0.1339 & 0.23201 & 0.00174 & 2614 & 32 & 2896 & 16 & 3064 & 22 \\
\hline ap05i11 & 0.1769 & 0.00115 & 1.8844 & 0.0249 & 0.07727 & 0.00104 & 1051 & 14 & 1076 & 18 & 1128 & 54 \\
\hline ap05i12 & 0.0732 & 0.00063 & 0.605 & 0.0097 & 0.05995 & 0.00098 & 453 & 9 & 480 & 12 & 600 & 72 \\
\hline ap05i13core & 0.1549 & 0.0009 & 1.5343 & 0.023 & 0.07183 & 0.00119 & 924 & 11 & 944 & 18 & 980 & 68 \\
\hline ap05i14rim & 0.0875 & 0.00044 & 0.7191 & 0.0065 & 0.05964 & 0.00052 & 541 & 5 & 550 & 8 & 590 & 38 \\
\hline ap05i15 & 0.1966 & 0.00077 & 2.1807 & 0.0177 & 0.08047 & 0.00058 & 1156 & 9 & 1175 & 11 & 1208 & 28 \\
\hline ap05i16 & 0.0909 & 0.00035 & 0.7429 & 0.0079 & 0.05929 & 0.00065 & 562 & 4 & 564 & 9 & 576 & 48 \\
\hline ap05j05 & 0.3835 & 0.0013 & 6.8902 & 0.0496 & 0.13033 & 0.00083 & 2092 & 13 & 2097 & 13 & 2102 & 22 \\
\hline ap05j06 & 0.1625 & 0.00065 & 1.8017 & 0.0092 & 0.08042 & 0.00039 & 971 & 8 & 1046 & 7 & 1206 & 20 \\
\hline ap05j07 & 0.177 & 0.00074 & 1.8419 & 0.0151 & 0.07546 & 0.00057 & 1052 & 9 & 1061 & 11 & 1080 & 30 \\
\hline ap05j08 & 0.1283 & 0.00059 & 1.2004 & 0.0096 & 0.06786 & 0.00058 & 771 & 7 & 801 & 9 & 864 & 34 \\
\hline ap05j09 & 0.0876 & 0.00084 & 0.7361 & 0.011 & 0.06093 & 0.00101 & 535 & 10 & 560 & 13 & 636 & 70 \\
\hline ap05j10 & 0.2448 & 0.00118 & 3.5992 & 0.0328 & 0.10666 & 0.00094 & 1399 & 13 & 1549 & 14 & 1742 & 32 \\
\hline
\end{tabular}


Table 1 (continued)

\begin{tabular}{|c|c|c|c|c|c|c|c|c|c|c|c|c|}
\hline Sample/analysis & ${ }^{206} \mathrm{~Pb} /{ }^{238} \mathrm{U}$ & $\begin{array}{l}\text { Percentage } \\
\text { error }\end{array}$ & ${ }^{207} \mathrm{~Pb} /{ }^{235} \mathrm{U}$ & $\begin{array}{l}\text { Percentage } \\
\text { error }\end{array}$ & ${ }^{207} \mathrm{~Pb} /{ }^{206} \mathrm{~Pb}$ & $\begin{array}{l}\text { Percentage } \\
\text { error }\end{array}$ & $\begin{array}{l}\text { Age, Ma } \\
{ }^{206} \mathrm{~Pb} /{ }^{238} \mathrm{U}\end{array}$ & $(2 \sigma)$ & ${ }^{207} \mathrm{~Pb} /{ }^{235} \mathrm{U}$ & $(2 \sigma)$ & ${ }^{207} \mathrm{~Pb} /{ }^{206} \mathrm{~Pb}$ & $(2 \sigma)$ \\
\hline \multicolumn{13}{|l|}{ HUERTA-1 } \\
\hline ap05j11 & 0.0907 & 0.00079 & 0.6987 & 0.0234 & 0.05588 & 0.0018 & 561 & 11 & 538 & 28 & 446 & 142 \\
\hline ap05j12 & 0.0908 & 0.00047 & 0.7568 & 0.0104 & 0.06045 & 0.00081 & 560 & 6 & 572 & 12 & 618 & 58 \\
\hline ap $05 j 13$ core & 0.108 & 0.00048 & 0.8978 & 0.0086 & 0.06027 & 0.00064 & 661 & 6 & 651 & 9 & 612 & 46 \\
\hline ap05j14rim & 0.101 & 0.00051 & 0.8343 & 0.007 & 0.0599 & 0.00053 & 619 & 6 & 616 & 8 & 598 & 38 \\
\hline ap05j15 & 0.1678 & 0.00057 & 1.7281 & 0.0137 & 0.07468 & 0.00062 & 999 & 7 & 1019 & 10 & 1058 & 34 \\
\hline ap05j16 & 0.0949 & 0.0005 & 0.7955 & 0.0086 & 0.06077 & 0.00067 & 584 & 6 & 594 & 10 & 630 & 48 \\
\hline
\end{tabular}

(iii) a tectonothermal event during the Jurassic that may be related to a mantle plume (Keppie et al., 2004b);

(iv) deposition of Mesozoic platformal rocks and Cenozoic arc-related rocks.

Our reexamination of the Xayacatlán area involving mapping and zircon geochronology shows that: (i) whereas some of the units are Ordovician or older others are post-Devonian (probably Carboniferous and Permian); (ii) high grade (amphibolite facies) metamorphism is restricted to syntectonic gabbroic intrusions and their contact aureoles intruded during transtensional deformation (none of the rocks have undergone eclogite facies metamorphism); and (iii) most of the N-S striking contacts between the units are vertical shear zones (not thrusts as previously inferred). These data are then applied to paleogeographic reconstructions and we suggest that whereas the Ordovician rocks formed on the southern margin of the Rheic Ocean, the Carboniferous and Permian rocks formed on the active Pacific margin of Pangea.

\section{Xayacatlán area}

The Xayacatlán area was originally mapped by OrtegaGutiérrez (1975), who recognized two units: retrograded eclogites of the Xayacatlán Formation that was thrust over the low grade Cosoltepec Formation (Fig. 2A). The Xayacatlán Formation was subsequently included in the eclogitic Piaxtla Group (RamírezEspinosa, 2001) and renamed the Piaxtla Suite by Middleton et al. (2007). However, the absence of eclogite facies metamorphism in the Xayacatlán area makes its inclusion in the Piaxtla Suite uncertain. Furthermore, correlation of the low grade rocks in the vicinity of Xayacatlán with the type Cosoltepec Formation are also uncertain because detrital zircon suites have different ages (c.f. Talavera-Mendoza et al., 2005; Keppie et al., 2006). In view of these problems, we define new names for most of the units in the Xayacatlán area (Figs. 2B and 3). The structure, metamorphism and geochemistry of the various units forms part of separate papers (e.g. Keppie et al., 2008b).

\subsection{Huerta Unit (new name)}

The Huerta Unit occurs with a N-S trending fault block that is bounded on its western side by sub-greenschist facies rocks assigned to the Tecomate Formation, and on its eastern side by rocks of the Amate Unit where the contact is a dextral shear zone formed under greenschist facies metamorphic conditions (Fig. 3). A megacrystic granite is exposed along the northern margin of the Xayacatlán area, however, its contact with the Huerta Unit is not exposed. It is composed of interbedded metapsammites and metapelites consisting of quartz, muscovite, chlorite and accessory zircon and opaque minerals. The unit is pervasively intruded by veins that gradually change in mineralogy from quartz veins in the west to pegmatites in the east. All of these rocks have undergone intense polyphase deformation at least three sets of folds including sheath folds under greenschist facies metamorphic conditions (Malone et al., 2002; and paper in preparation by authors). In view of this, it is impossible to measure a type section. However, the road between Xayacatlán and Acatlán provides an excellent cross-section of the unit. A sample from the western part of this unit has yielded detrital zircons, the youngest of which yielded a concordant age of $455 \pm 4 \mathrm{Ma}$ with older population age peaks at $\sim 600 \mathrm{Ma}$ and $\sim 1-1.2 \mathrm{Ga}$ (Keppie et al., 2006). A pegmatite sample (Fig. 3: HUERTA-1: $18^{\circ} 14.758^{\prime}, 97^{\circ} 58.727^{\prime}$ ) was collected in the eastern part of the fault block for $\mathrm{U}-\mathrm{Pb}$ detrital zircon analysis (Fig. 3).

\subsection{Amate Unit (new name)}

The Amate Unit occurs in the central N-S trending fault block (Fig. 3). All the contacts with other rock units are dextral shear zones. The Amate Unit is mainly composed of metaarkose, metapsammite, and metapelite that are intruded by mafic and felsic dikes. The metasedimentary rocks generally consist of quartz, muscovite, feldspar, and chlorite with accessory zircon and opaque minerals. However, adjacent to the largest mafic dike, the rocks are hornfelsed and contain large decussate muscovite and clinozoisite crystals. The petrography, geochemistry and geochronology of the mafic (gabbro and diabase) and felsic (granite and pegmatite) rocks have been described by Keppie et al. (2008b). They are mainly mafic amphibolitic gneisses with ultramafic and anorthositic bands with a continental tholeiitic signature cut by medium grained mafic dikes and granitic pegmatites. In places, these mafic dikes have undergone intense shearing associated with retrogressive metamorphism that produced serpentinite and chlorite schist. The rocks of the Amate Unit have been deformed by at least three sets of folds generally developed under greenschist facies metamorphic conditions (detailed in a paper in preparation by authors). As a 
Table 2

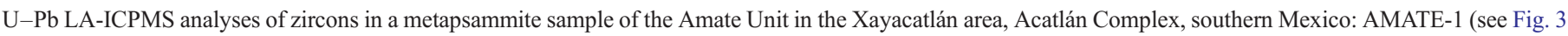
for sample locations)

\begin{tabular}{|c|c|c|c|c|c|c|c|c|c|c|c|c|}
\hline Sample/analysis & ${ }^{206} \mathrm{~Pb} /{ }^{238} \mathrm{U}$ & $\begin{array}{l}\text { Percentage } \\
\text { error }\end{array}$ & ${ }^{207} \mathrm{~Pb} /{ }^{235} \mathrm{U}$ & $\begin{array}{l}\text { Percentage } \\
\text { error }\end{array}$ & ${ }^{207} \mathrm{~Pb} /{ }^{206} \mathrm{~Pb}$ & $\begin{array}{l}\text { Percentage } \\
\text { error }\end{array}$ & $\begin{array}{l}\text { Age, Ma } \\
{ }^{206} \mathrm{~Pb} /{ }^{238} \mathrm{U}\end{array}$ & $(2 \sigma)$ & ${ }^{207} \mathrm{~Pb} /{ }^{235} \mathrm{U}$ & $(2 \sigma)$ & ${ }^{207} \mathrm{~Pb} /{ }^{206} \mathrm{~Pb}$ & $(2 \sigma)$ \\
\hline \multicolumn{13}{|l|}{ AMATE-1 } \\
\hline ap03h05 & 0.1959 & 0.0007 & 2.1751 & 0.0126 & 0.08053 & 0.0005 & 1151 & 8 & 1173 & 8 & 1208 & 26 \\
\hline ap03h06 & 0.2068 & 0.0008 & 2.3453 & 0.0178 & 0.08225 & 0.0005 & 1211 & 9 & 1226 & 11 & 1250 & 26 \\
\hline ap03h07 & 0.151 & 0.0005 & 1.4568 & 0.0079 & 0.06998 & 0.0003 & 906 & 6 & 913 & 6 & 926 & 18 \\
\hline ap03h08 & 0.2138 & 0.0011 & 2.4633 & 0.0227 & 0.08357 & 0.0009 & 1248 & 12 & 1261 & 13 & 1282 & 40 \\
\hline ap03h09 & 0.1692 & 0.0009 & 1.767 & 0.0186 & 0.07573 & 0.0008 & 1006 & 10 & 1033 & 14 & 1088 & 42 \\
\hline ap03h10 & 0.1823 & 0.0011 & 1.8743 & 0.0337 & 0.07456 & 0.0013 & 1079 & 12 & 1072 & 24 & 1056 & 70 \\
\hline ap03h11 & 0.1542 & 0.0018 & 1.4552 & 0.0469 & 0.06845 & 0.0025 & 923 & 21 & 912 & 39 & 882 & 154 \\
\hline ap03h12 & 0.1664 & 0.0007 & 1.6448 & 0.0176 & 0.07169 & 0.0008 & 992 & 8 & 988 & 13 & 976 & 44 \\
\hline ap03h13 & 0.1592 & 0.0006 & 1.5677 & 0.0121 & 0.07142 & 0.0005 & 953 & 8 & 958 & 10 & 968 & 28 \\
\hline ap03h14 & 0.2091 & 0.0017 & 2.4126 & 0.0396 & 0.0837 & 0.0012 & 1222 & 18 & 1246 & 24 & 1284 & 56 \\
\hline ap03h15 & 0.1965 & 0.0008 & 2.1623 & 0.0132 & 0.07982 & 0.0006 & 1157 & 9 & 1169 & 8 & 1192 & 28 \\
\hline ap03h16 & 0.1589 & 0.0006 & 1.683 & 0.0076 & 0.07679 & 0.0003 & 952 & 7 & 1002 & 6 & 1114 & 16 \\
\hline ap03i05 & 0.1716 & 0.0009 & 1.7676 & 0.017 & 0.0747 & 0.0007 & 1021 & 10 & 1034 & 12 & 1060 & 36 \\
\hline ap03i06 & 0.1805 & 0.0008 & 1.8891 & 0.0134 & 0.0759 & 0.0004 & 1069 & 9 & 1077 & 9 & 1092 & 24 \\
\hline ap03i07 & 0.1723 & 0.0007 & 1.7778 & 0.0135 & 0.07482 & 0.0006 & 1025 & 8 & 1037 & 10 & 1062 & 30 \\
\hline ap03i08 & 0.1642 & 0.0022 & 1.908 & 0.0277 & 0.08429 & 0.0004 & 978 & 26 & 1084 & 19 & 1298 & 20 \\
\hline ap03i09 & 0.1902 & 0.0012 & 2.1003 & 0.0269 & 0.0801 & 0.0011 & 1120 & 15 & 1149 & 18 & 1198 & 56 \\
\hline ap03i10 & 0.1501 & 0.0012 & 1.4315 & 0.0269 & 0.06916 & 0.0013 & 900 & 14 & 902 & 22 & 902 & 78 \\
\hline ap03i11 & 0.1411 & 0.0006 & 1.3589 & 0.0121 & 0.06987 & 0.0007 & 847 & 7 & 871 & 10 & 924 & 38 \\
\hline ap03i12 & 0.195 & 0.0013 & 2.1476 & 0.0288 & 0.07987 & 0.0011 & 1147 & 15 & 1164 & 19 & 1192 & 54 \\
\hline ap03i13 & 0.1945 & 0.0007 & 2.1055 & 0.0116 & 0.07849 & 0.0004 & 1147 & 7 & 1151 & 8 & 1158 & 20 \\
\hline ap03i14 & 0.2046 & 0.0014 & 2.2091 & 0.0258 & 0.07832 & 0.0009 & 1205 & 15 & 1184 & 16 & 1154 & 48 \\
\hline ap03i15 & 0.1725 & 0.0006 & 1.7495 & 0.0121 & 0.07357 & 0.0005 & 1027 & 6 & 1027 & 9 & 1028 & 26 \\
\hline ap03i16 & 0.2112 & 0.0009 & 2.4046 & 0.0137 & 0.08257 & 0.0005 & 1235 & 9 & 1244 & 8 & 1258 & 24 \\
\hline ap03j05 & 0.2149 & 0.0012 & 2.3879 & 0.0224 & 0.08061 & 0.0008 & 1256 & 13 & 1239 & 14 & 1210 & 38 \\
\hline ap03j06 & 0.1877 & 0.0008 & 2.0741 & 0.0091 & 0.08015 & 0.0003 & 1111 & 8 & 1140 & 6 & 1200 & 14 \\
\hline ap03j07 & 0.2015 & 0.0007 & 2.2795 & 0.0125 & 0.08206 & 0.0004 & 1180 & 8 & 1206 & 8 & 1246 & 20 \\
\hline ap03j08 & 0.1536 & 0.0008 & 1.61 & 0.0079 & 0.076 & 0.0003 & 924 & 10 & 974 & 6 & 1094 & 20 \\
\hline ap03j09 & 0.156 & 0.0011 & 1.5346 & 0.0273 & 0.07136 & 0.0014 & 933 & 13 & 944 & 22 & 966 & 78 \\
\hline ap03j10 & 0.2282 & 0.0009 & 2.7648 & 0.0182 & 0.08789 & 0.0006 & 1325 & 9 & 1346 & 10 & 1380 & 26 \\
\hline ap03j11 & 0.1699 & 0.0007 & 1.7146 & 0.0111 & 0.0732 & 0.0006 & 1013 & 8 & 1014 & 8 & 1018 & 32 \\
\hline ap03j12 & 0.1724 & 0.0009 & 1.8003 & 0.0247 & 0.07573 & 0.0011 & 1025 & 10 & 1046 & 18 & 1086 & 58 \\
\hline ap03j13 & 0.2011 & 0.0007 & 2.1895 & 0.0204 & 0.07898 & 0.0008 & 1182 & 8 & 1178 & 13 & 1170 & 40 \\
\hline ap03j14 & 0.1894 & 0.002 & 2.0413 & 0.0274 & 0.07818 & 0.0008 & 1116 & 22 & 1129 & 18 & 1150 & 40 \\
\hline ap03j15 & 0.1698 & 0.0005 & 1.7364 & 0.0099 & 0.07417 & 0.0004 & 1011 & 6 & 1022 & 7 & 1046 & 22 \\
\hline ap03j16 & 0.1585 & 0.0011 & 1.5431 & 0.0201 & 0.07064 & 0.0009 & 948 & 12 & 948 & 16 & 946 & 50 \\
\hline ap03k05 & 0.1932 & 0.0007 & 2.1011 & 0.0118 & 0.07887 & 0.0004 & 1138 & 8 & 1149 & 8 & 1168 & 20 \\
\hline ap03k06 & 0.1832 & 0.0008 & 1.9052 & 0.0105 & 0.07543 & 0.0005 & 1084 & 9 & 1083 & 7 & 1078 & 26 \\
\hline ap03k07 & 0.156 & 0.0009 & 1.5089 & 0.0243 & 0.07017 & 0.0013 & 936 & 11 & 934 & 20 & 932 & 76 \\
\hline ap03k08 & 0.1702 & 0.0012 & 1.7423 & 0.0211 & 0.07427 & 0.0008 & 1013 & 14 & 1024 & 16 & 1048 & 44 \\
\hline ap03k09 & 0.1854 & 0.0008 & 1.9556 & 0.0198 & 0.07651 & 0.0008 & 1098 & 9 & 1100 & 14 & 1108 & 42 \\
\hline ap03k10 & 0.1921 & 0.0008 & 2.0666 & 0.0118 & 0.07803 & 0.0004 & 1134 & 9 & 1138 & 8 & 1146 & 22 \\
\hline ap03k11 & 0.1968 & 0.0006 & 2.4169 & 0.0126 & 0.0891 & 0.0003 & 1151 & 7 & 1248 & 7 & 1406 & 14 \\
\hline ap03k12 & 0.1721 & 0.0007 & 1.7297 & 0.0164 & 0.0729 & 0.0007 & 1024 & 8 & 1020 & 12 & 1010 & 40 \\
\hline ap03k13 & 0.1743 & 0.0005 & 1.7838 & 0.01 & 0.07421 & 0.0004 & 1036 & 6 & 1040 & 7 & 1046 & 20 \\
\hline ap03k14 & 0.1678 & 0.0007 & 1.6805 & 0.0096 & 0.07264 & 0.0004 & 1000 & 7 & 1001 & 7 & 1004 & 22 \\
\hline ap03k15 & 0.1896 & 0.0009 & 2.1294 & 0.023 & 0.08147 & 0.0009 & 1116 & 10 & 1158 & 15 & 1232 & 46 \\
\hline ap03k16 & 0.2207 & 0.0009 & 2.6476 & 0.0191 & 0.08701 & 0.0006 & 1285 & 10 & 1314 & 11 & 1360 & 24 \\
\hline ap03105 & 0.1697 & 0.001 & 1.7163 & 0.0156 & 0.07335 & 0.0006 & 1010 & 11 & 1015 & 12 & 1022 & 34 \\
\hline ap03106 & 0.1762 & 0.0011 & 1.8235 & 0.0352 & 0.07506 & 0.0015 & 1049 & 14 & 1054 & 25 & 1070 & 82 \\
\hline ap03107 & 0.1596 & 0.0008 & 1.6017 & 0.0178 & 0.07277 & 0.0008 & 951 & 10 & 971 & 14 & 1006 & 48 \\
\hline ap03108 & 0.1578 & 0.0006 & 1.5676 & 0.0152 & 0.07207 & 0.0008 & 945 & 7 & 957 & 12 & 986 & 42 \\
\hline ap03109 & 0.1877 & 0.0008 & 2.187 & 0.0103 & 0.08451 & 0.0004 & 1105 & 10 & 1177 & 7 & 1304 & 16 \\
\hline ap03110 & 0.2089 & 0.0008 & 2.3823 & 0.0157 & 0.08273 & 0.0006 & 1223 & 9 & 1237 & 9 & 1262 & 26 \\
\hline ap03111 & 0.2148 & 0.0009 & 2.5011 & 0.0133 & 0.08446 & 0.0004 & 1251 & 11 & 1272 & 8 & 1302 & 20 \\
\hline ap03112 & 0.1756 & 0.0008 & 1.7631 & 0.0153 & 0.07282 & 0.0006 & 1043 & 9 & 1032 & 11 & 1008 & 32 \\
\hline ap03113 & 0.1649 & 0.0016 & 1.6489 & 0.0424 & 0.07253 & 0.002 & 985 & 19 & 989 & 32 & 1000 & 110 \\
\hline ap03114 & 0.2224 & 0.0009 & 3.5857 & 0.0384 & 0.11693 & 0.0012 & 1243 & 15 & 1546 & 17 & 1908 & 36 \\
\hline ap03115 & 0.158 & 0.001 & 1.5318 & 0.0201 & 0.07031 & 0.0008 & 946 & 12 & 943 & 16 & 936 & 48 \\
\hline ap03116 & 0.143 & 0.001 & 1.4067 & 0.0255 & 0.07133 & 0.0013 & 856 & 12 & 892 & 22 & 966 & 78 \\
\hline
\end{tabular}


Table 2 (continued)

\begin{tabular}{|c|c|c|c|c|c|c|c|c|c|c|c|c|}
\hline Sample/analysis & ${ }^{206} \mathrm{~Pb} /{ }^{238} \mathrm{U}$ & $\begin{array}{l}\text { Percentage } \\
\text { error }\end{array}$ & ${ }^{207} \mathrm{~Pb} /{ }^{235} \mathrm{U}$ & $\begin{array}{l}\text { Percentage } \\
\text { error }\end{array}$ & ${ }^{207} \mathrm{~Pb} /{ }^{206} \mathrm{~Pb}$ & $\begin{array}{l}\text { Percentage } \\
\text { error }\end{array}$ & $\begin{array}{l}\text { Age, Ma } \\
{ }^{206} \mathrm{~Pb} /{ }^{238} \mathrm{U}\end{array}$ & $(2 \sigma)$ & ${ }^{207} \mathrm{~Pb} /{ }^{235} \mathrm{U}$ & $(2 \sigma)$ & ${ }^{207} \mathrm{~Pb} /{ }^{206} \mathrm{~Pb}$ & $(2 \sigma)$ \\
\hline \multicolumn{13}{|l|}{ AMATE-1 } \\
\hline ap03m05 & 0.1631 & 0.0007 & 1.6395 & 0.009 & 0.07289 & 0.0003 & 974 & 8 & 986 & 7 & 1010 & 16 \\
\hline ap03m06 & 0.1874 & 0.0014 & 1.9049 & 0.037 & 0.07371 & 0.0014 & 1106 & 16 & 1083 & 26 & 1032 & 76 \\
\hline ap03m07 & 0.1751 & 0.0012 & 1.7998 & 0.0149 & 0.07455 & 0.0008 & 1040 & 14 & 1045 & 11 & 1056 & 46 \\
\hline ap03m08 & 0.1663 & 0.0008 & 1.7034 & 0.0121 & 0.07428 & 0.0005 & 988 & 10 & 1010 & 9 & 1048 & 28 \\
\hline Ap03m09 & 0.2021 & 0.0012 & 2.1879 & 0.0234 & 0.07851 & 0.0008 & 1187 & 13 & 1177 & 15 & 1158 & 42 \\
\hline Ap03m10 & 0.2232 & 0.0008 & 2.5847 & 0.0194 & 0.08398 & 0.0006 & 1299 & 9 & 1296 & 11 & 1292 & 26 \\
\hline Ap03m11 & 0.2099 & 0.0011 & 2.3755 & 0.0283 & 0.08207 & 0.0009 & 1228 & 12 & 1235 & 17 & 1246 & 44 \\
\hline Ap03m12 & 0.1701 & 0.0007 & 1.7071 & 0.0142 & 0.0728 & 0.0007 & 1012 & 9 & 1011 & 11 & 1008 & 38 \\
\hline Ap03m13 & 0.2032 & 0.0009 & 2.2199 & 0.0173 & 0.07925 & 0.0007 & 1192 & 10 & 1187 & 11 & 1178 & 38 \\
\hline Ap03m14 & 0.2012 & 0.001 & 2.1713 & 0.0154 & 0.07826 & 0.0005 & 1184 & 12 & 1172 & 10 & 1152 & 28 \\
\hline Ap03m15 & 0.1607 & 0.0014 & 1.5535 & 0.0259 & 0.0701 & 0.0014 & 963 & 17 & 952 & 21 & 930 & 80 \\
\hline Ap03m16 & 0.2001 & 0.0009 & 2.228 & 0.0118 & 0.08077 & 0.0004 & 1175 & 11 & 1190 & 7 & 1214 & 18 \\
\hline Ap03n05 & 0.2141 & 0.001 & 2.4366 & 0.0239 & 0.08255 & 0.0008 & 1252 & 12 & 1253 & 14 & 1258 & 36 \\
\hline Ap03n06 & 0.1834 & 0.0007 & 1.955 & 0.0174 & 0.07732 & 0.0007 & 1082 & 9 & 1100 & 12 & 1128 & 34 \\
\hline Ap03n07 & 0.1494 & 0.0006 & 1.5046 & 0.0129 & 0.07302 & 0.0006 & 896 & 8 & 932 & 10 & 1014 & 34 \\
\hline Ap03n08 & 0.2004 & 0.001 & 2.2346 & 0.0183 & 0.08087 & 0.0006 & 1176 & 11 & 1192 & 11 & 1218 & 30 \\
\hline Ap03n09 & 0.1472 & 0.0005 & 1.471 & 0.0078 & 0.07246 & 0.0004 & 884 & 6 & 919 & 6 & 998 & 24 \\
\hline Ap03n10 & 0.1924 & 0.0009 & 2.1696 & 0.0165 & 0.08178 & 0.0006 & 1120 & 12 & 1171 & 11 & 1240 & 28 \\
\hline Ap03n11 & 0.1547 & 0.0011 & 1.5071 & 0.0213 & 0.07064 & 0.001 & 926 & 13 & 933 & 17 & 946 & 56 \\
\hline Ap03n12 & 0.173 & 0.0007 & 1.7662 & 0.014 & 0.07404 & 0.0005 & 1029 & 9 & 1033 & 10 & 1042 & 30 \\
\hline Ap03n13 & 0.2089 & 0.0008 & 2.3261 & 0.017 & 0.08074 & 0.0006 & 1221 & 9 & 1220 & 10 & 1214 & 28 \\
\hline Ap03n14 & 0.1688 & 0.0012 & 1.6488 & 0.0251 & 0.07085 & 0.0013 & 1006 & 14 & 989 & 19 & 952 & 78 \\
\hline Ap03n15 & 0.1709 & 0.0007 & 1.7168 & 0.011 & 0.07285 & 0.0005 & 1017 & 9 & 1015 & 8 & 1008 & 26 \\
\hline Ap03n16 & 0.1749 & 0.001 & 1.7606 & 0.0178 & 0.07302 & 0.0009 & 1039 & 12 & 1031 & 13 & 1014 & 50 \\
\hline
\end{tabular}

consequence of the polyphase deformation, no stratigraphic section is available for a type section: the Amate River provides the best section of the unit (Fig. 3). Three samples were collected for $\mathrm{U}-\mathrm{Pb}$ zircon analyses (Fig. 3): one from a feldspathic psammite (AMATE-1, $18^{\circ} 13.793^{\prime}, 97^{\circ} 58.965^{\prime}$ ), and the other two from cross-cutting foliated granitic dikes (AMATE-2: $18^{\circ}$ $13.772^{\prime}, 97^{\circ} 58.963^{\prime}$; SAL-12: $\left.18^{\circ} 13.247^{\prime}, 97^{\circ} 57.608^{\prime}\right)$.

\subsection{Salada Unit (new name)}

The Salada Unit occurs in a N-S trending fault block in the eastern part of the area. It is bounded on its western side by a dextral shear zone that juxtaposes it against the Tecomate Formation, on its northern side by an E-W trending, shallow $\mathrm{N}$-dipping shear zone, above which lies the Tecomate Formation, and to the south it is unconformably overlain by Cenozoic rocks (Fig. 3). It consists mainly of metapelites, metapsammites and thin, tectonically intercalated mafic lenses. The metasedimentary rocks made up of quartz, muscovite, chlorite and accessory opaque minerals. The mafic rocks are composed of amphibole, chlorite, feldspar, epidote and accessory opaque minerals. A metapsammite sample was collected for $\mathrm{U}-\mathrm{Pb}$ zircon analysis (Fig. 3: SAL-11: $18^{\circ} 14.077^{\prime}, 97^{\circ} 57.341^{\prime}$ ).

\subsection{Tecomate Formation}

The Tecomate Formation in the Xayacatán area is tectonically juxtaposed against all the previously described units, either along $\mathrm{N}-\mathrm{S}$ trending dextral shear zones or above gently
$\mathrm{N}$-dipping shear zones with gently N-plunging stretching lineations (Malone et al., 2002; paper in preparation by authors) (Fig. 3). In the north, it is overlain unconformably by Mesozoic rocks, whereas in the southeast it is faulted against the Totoltepec laccolith (Fig. 3). The Tecomate Formation consists predominantly of meta-arkoses, metapsammites, slates, metaconglomerates, and marbles. The clastic rocks consist mainly of quartz, feldspar, muscovite, chlorite, epidote and opaque minerals. The conglomerates contain a diverse assemblage of pebbles: granitoid and volcanic rocks, metapsammite, and vein quartz. Granitoid pebbles from a conglomerate $\sim 20 \mathrm{~km}$ to the east of the Xayacatlán area have yielded U-Pb ages of $\sim 290 \mathrm{Ma}$ and are inferred to have been derived from the Lower Permian Totoltepec laccolith (Yañez et al., 1991; Keppie et al., 2004b), which is exposed along the eastern margin of Fig. 3. On the other hand, marbles in the type section of the Tecomate Formation exposed to the south of the town of Acatlán (Fig. 1B), have yielded latest Pennsylvanian-Early Permian microfossils (Keppie et al., 2004a). Deformation in the Tecomate Formation in the Xayacatlán area varies from almost undeformed to polyphase involving three sets of folds (Malone et al., 2002; and paper in preparation by authors). The grade of metamorphism varies from sub-greenschist facies to lower greenschist facies (Malone et al., 2002).

\subsection{Totoltepec laccolith}

The Totoltepec laccolith crops out along the eastern margin of the Xayacatlán area (Fig. 3). It has yielded concordant $\mathrm{U}-\mathrm{Pb}$ zircon ages of $\sim 288 \mathrm{Ma}$ (Yañez et al. et al., 1991; Keppie et al., 
Table 3

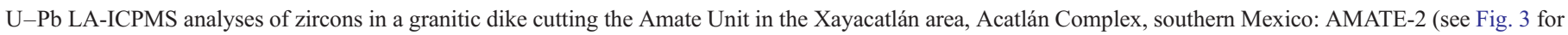
sample locations)

\begin{tabular}{|c|c|c|c|c|c|c|c|c|c|c|c|c|}
\hline Sample/analysis & ${ }^{206} \mathrm{~Pb} /{ }^{238} \mathrm{U}$ & $\begin{array}{l}\text { Percentage } \\
\text { error }\end{array}$ & ${ }^{207} \mathrm{~Pb} /{ }^{235} \mathrm{U}$ & $\begin{array}{l}\text { Percentage } \\
\text { error }\end{array}$ & ${ }^{207} \mathrm{~Pb} /{ }^{206} \mathrm{~Pb}$ & $\begin{array}{l}\text { Percentage } \\
\text { error }\end{array}$ & $\begin{array}{l}\text { Age, Ma } \\
{ }^{206} \mathrm{~Pb} /{ }^{238} \mathrm{U}\end{array}$ & $(2 \sigma)$ & ${ }^{207} \mathrm{~Pb} /{ }^{235} \mathrm{U}$ & $(2 \sigma)$ & ${ }^{207} \mathrm{~Pb} /{ }^{206} \mathrm{~Pb}$ & $(2 \sigma)$ \\
\hline \multicolumn{13}{|l|}{$A M A T E-2$} \\
\hline Ap05k05 & 0.0737 & 0.0006 & 0.5643 & 0.013 & 0.05554 & 0.00118 & 457 & 8 & 454 & 17 & 434 & 94 \\
\hline Ap05k06 & 0.2138 & 0.0014 & 2.5109 & 0.0271 & 0.0852 & 0.00085 & 1252 & 17 & 1275 & 16 & 1318 & 38 \\
\hline Ap05k07 & 0.0745 & 0.0005 & 0.5894 & 0.0136 & 0.05741 & 0.00134 & 462 & 7 & 470 & 17 & 506 & 104 \\
\hline Ap05k08 & 0.0717 & 0.0002 & 0.5543 & 0.0022 & 0.05606 & 0.0002 & 447 & 3 & 448 & 3 & 454 & 16 \\
\hline Ap05k09 & 0.071 & 0.0002 & 0.5598 & 0.0029 & 0.05721 & 0.00027 & 444 & 3 & 451 & 4 & 498 & 20 \\
\hline ap05k10 & 0.0808 & 0.0004 & 0.74 & 0.0081 & 0.06646 & 0.00073 & 495 & 5 & 562 & 10 & 820 & 46 \\
\hline ap05k11 & 0.1871 & 0.0007 & 2.0692 & 0.0174 & 0.08023 & 0.00054 & 1102 & 8 & 1139 & 11 & 1202 & 26 \\
\hline ap05k12 & 0.0702 & 0.0004 & 0.525 & 0.0104 & 0.05427 & 0.00112 & 437 & 6 & 428 & 14 & 382 & 92 \\
\hline ap05k13 & 0.071 & 0.0006 & 0.5561 & 0.018 & 0.05677 & 0.00194 & 442 & 8 & 449 & 23 & 482 & 152 \\
\hline ap05k14 & 0.2115 & 0.0013 & 2.3549 & 0.0162 & 0.08077 & 0.00058 & 1235 & 15 & 1229 & 10 & 1214 & 30 \\
\hline ap $05 \mathrm{k} 15$ & 0.2128 & 0.0008 & 2.4141 & 0.0176 & 0.08227 & 0.00064 & 1244 & 9 & 1247 & 11 & 1250 & 30 \\
\hline ap $05 \mathrm{k} 16$ & 0.1794 & 0.0012 & 1.838 & 0.0298 & 0.07432 & 0.00115 & 1061 & 14 & 1059 & 21 & 1050 & 62 \\
\hline ap05105 & 0.1579 & 0.0012 & 1.5687 & 0.0207 & 0.07205 & 0.00104 & 944 & 14 & 958 & 16 & 986 & 60 \\
\hline ap05106 & 0.192 & 0.0013 & 2.0882 & 0.0232 & 0.0789 & 0.00082 & 1132 & 15 & 1145 & 15 & 1168 & 40 \\
\hline ap05107 & 0.1708 & 0.0006 & 1.8449 & 0.0116 & 0.07833 & 0.00052 & 1018 & 7 & 1062 & 8 & 1154 & 26 \\
\hline ap05108 & 0.072 & 0.0003 & 0.6143 & 0.0045 & 0.0619 & 0.00048 & 447 & 4 & 486 & 6 & 670 & 32 \\
\hline ap05109 & 0.0723 & 0.0003 & 0.5715 & 0.0034 & 0.05729 & 0.00037 & 449 & 3 & 459 & 4 & 502 & 28 \\
\hline ap05110 & 0.1523 & 0.001 & 1.5168 & 0.0155 & 0.07226 & 0.00059 & 914 & 12 & 937 & 13 & 992 & 32 \\
\hline ap05111 & 0.1588 & 0.0008 & 1.5635 & 0.0152 & 0.07139 & 0.00066 & 950 & 9 & 956 & 12 & 968 & 38 \\
\hline ap05112 & 0.0734 & 0.0004 & 0.5574 & 0.0093 & 0.05505 & 0.00088 & 458 & 5 & 450 & 12 & 414 & 70 \\
\hline ap05113 & 0.0709 & 0.0005 & 0.5503 & 0.0144 & 0.05628 & 0.00154 & 442 & 7 & 445 & 19 & 462 & 122 \\
\hline ap05114 & 0.0693 & 0.0005 & 0.5402 & 0.0077 & 0.05653 & 0.00083 & 432 & 6 & 439 & 10 & 472 & 64 \\
\hline ap05115 & 0.1225 & 0.0006 & 1.187 & 0.0094 & 0.07028 & 0.00067 & 745 & 7 & 795 & 9 & 936 & 38 \\
\hline ap05116 & 0.073 & 0.0006 & 0.6545 & 0.0107 & 0.06506 & 0.0007 & 451 & 7 & 511 & 13 & 776 & 46 \\
\hline ap $05 \mathrm{~m} 05$ & 0.0706 & 0.0006 & 0.5539 & 0.013 & 0.05688 & 0.0014 & 440 & 8 & 448 & 17 & 486 & 110 \\
\hline ap05m06rim & 0.0744 & 0.0002 & 0.5863 & 0.0029 & 0.05713 & 0.00026 & 462 & 3 & 469 & 4 & 496 & 20 \\
\hline ap $05 \mathrm{~m} 07$ core & 0.1217 & 0.0005 & 1.118 & 0.0111 & 0.06662 & 0.00061 & 740 & 6 & 762 & 11 & 824 & 38 \\
\hline ap05m08 & 0.1343 & 0.0006 & 1.2545 & 0.0133 & 0.06777 & 0.00072 & 809 & 7 & 825 & 12 & 860 & 44 \\
\hline ap05m09 & 0.0764 & 0.0004 & 0.6006 & 0.0069 & 0.05702 & 0.00064 & 476 & 5 & 478 & 9 & 492 & 50 \\
\hline ap $05 \mathrm{~m} 10$ & 0.1674 & 0.0007 & 1.6782 & 0.0123 & 0.07272 & 0.00057 & 997 & 8 & 1000 & 9 & 1006 & 30 \\
\hline ap05m11 & 0.1959 & 0.0009 & 2.143 & 0.0238 & 0.07936 & 0.00084 & 1154 & 10 & 1163 & 15 & 1180 & 40 \\
\hline ap $05 \mathrm{~m} 12$ & 0.2101 & 0.0007 & 2.3772 & 0.0138 & 0.08205 & 0.00053 & 1230 & 8 & 1236 & 8 & 1246 & 26 \\
\hline ap05m13 & 0.0711 & 0.0004 & 0.5631 & 0.0091 & 0.05743 & 0.00097 & 442 & 5 & 454 & 12 & 508 & 74 \\
\hline ap $05 \mathrm{~m} 14$ & 0.0723 & 0.0005 & 0.5602 & 0.0103 & 0.05617 & 0.00112 & 450 & 6 & 452 & 13 & 458 & 88 \\
\hline ap $05 \mathrm{~m} 15$ & 0.1639 & 0.0006 & 1.6294 & 0.0116 & 0.0721 & 0.00055 & 978 & 7 & 982 & 9 & 988 & 32 \\
\hline ap $05 \mathrm{~m} 16$ & 0.2099 & 0.0009 & 2.3427 & 0.0225 & 0.08096 & 0.00075 & 1228 & 11 & 1225 & 14 & 1220 & 36 \\
\hline ap05n05 & 0.0279 & 0.0003 & 0.2063 & 0.0069 & 0.05365 & 0.00193 & 177 & 4 & 190 & 12 & 356 & 162 \\
\hline ap05n06 & 0.1935 & 0.0016 & 2.1068 & 0.0356 & 0.07898 & 0.00132 & 1140 & 19 & 1151 & 23 & 1170 & 66 \\
\hline ap05n07 & 0.1923 & 0.0013 & 2.1478 & 0.0247 & 0.08099 & 0.00081 & 1134 & 15 & 1164 & 16 & 1220 & 40 \\
\hline ap05n08 & 0.0694 & 0.0004 & 0.5338 & 0.0075 & 0.05582 & 0.00087 & 433 & 5 & 434 & 10 & 444 & 68 \\
\hline ap05n09 & 0.0697 & 0.0007 & 0.5297 & 0.0122 & 0.05515 & 0.00116 & 435 & 9 & 432 & 16 & 418 & 94 \\
\hline ap $05 n 10$ & 0.071 & 0.0004 & 0.5539 & 0.0089 & 0.05658 & 0.00094 & 442 & 6 & 448 & 12 & 474 & 74 \\
\hline ap05n11 & 0.0697 & 0.0004 & 0.524 & 0.0088 & 0.05455 & 0.00087 & 434 & 6 & 428 & 12 & 392 & 72 \\
\hline ap05n12core & 0.0705 & 0.0003 & 0.5318 & 0.006 & 0.0547 & 0.00066 & 439 & 4 & 433 & 8 & 400 & 54 \\
\hline ap05n13rim & 0.0711 & 0.0003 & 0.5513 & 0.004 & 0.05621 & 0.00043 & 443 & 4 & 446 & 5 & 460 & 34 \\
\hline ap05n14rim & 0.0749 & 0.0004 & 0.589 & 0.0074 & 0.05707 & 0.00074 & 466 & 5 & 470 & 9 & 494 & 58 \\
\hline ap $05 n 15$ core & 0.0709 & 0.0003 & 0.5573 & 0.0065 & 0.05705 & 0.00068 & 441 & 4 & 450 & 8 & 492 & 52 \\
\hline ap05n16 & 0.0962 & 0.0006 & 0.7973 & 0.0237 & 0.06012 & 0.00177 & 594 & 9 & 595 & 27 & 606 & 128 \\
\hline ap05o05 & 0.199 & 0.001 & 2.4302 & 0.0277 & 0.08856 & 0.00094 & 1163 & 12 & 1252 & 16 & 1394 & 40 \\
\hline ap05o06 & 0.1482 & 0.0014 & 1.387 & 0.0226 & 0.0679 & 0.00101 & 890 & 16 & 883 & 19 & 864 & 62 \\
\hline ap05o07 & 0.072 & 0.0003 & 0.5789 & 0.0042 & 0.05833 & 0.00043 & 447 & 4 & 464 & 5 & 542 & 32 \\
\hline ap05o08 & 0.1651 & 0.0009 & 1.6873 & 0.0202 & 0.07413 & 0.00089 & 985 & 11 & 1004 & 15 & 1044 & 48 \\
\hline ap05o09core & 0.1955 & 0.001 & 2.1556 & 0.0181 & 0.07998 & 0.00078 & 1151 & 11 & 1167 & 12 & 1196 & 38 \\
\hline ap05o10rim & 0.0725 & 0.0003 & 0.6449 & 0.0104 & 0.06451 & 0.00095 & 447 & 4 & 505 & 13 & 758 & 62 \\
\hline ap05o11core & 0.1599 & 0.0012 & 1.583 & 0.0258 & 0.07179 & 0.00116 & 954 & 15 & 964 & 20 & 978 & 66 \\
\hline ap05o12rim & 0.0742 & 0.0003 & 0.6091 & 0.0044 & 0.05957 & 0.00042 & 459 & 3 & 483 & 6 & 586 & 30 \\
\hline
\end{tabular}

2004b). It varies from mafic (hornblende, epidote, chlorite) to felsic (quartz, feldspar, muscovite) and has a calc-alkaline geochemistry interpreted in terms of arc magmatism (Malone et al., 2002). The laccolith contains a folded foliation with a $\mathrm{N}$-plunging mineral lineation interpreted in terms of syntectonic intrusion during S-vergent thrusting (Malone et al., 2002). 
Table 4

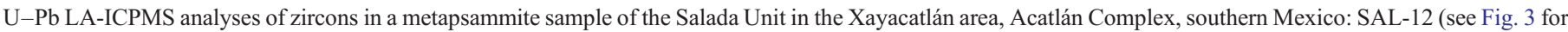
sample locations)

\begin{tabular}{|c|c|c|c|c|c|c|c|c|c|c|c|c|}
\hline Sample/analysis & ${ }^{206} \mathrm{~Pb} /{ }^{238} \mathrm{U}$ & $\begin{array}{l}\text { Percentage } \\
\text { error }\end{array}$ & ${ }^{207} \mathrm{~Pb} /{ }^{235} \mathrm{U}$ & $\begin{array}{l}\text { Percentage } \\
\text { error }\end{array}$ & ${ }^{207} \mathrm{~Pb} /{ }^{206} \mathrm{~Pb}$ & $\begin{array}{l}\text { Percentage } \\
\text { error }\end{array}$ & $\begin{array}{l}\text { Age, Ma } \\
{ }^{206} \mathrm{~Pb} /{ }^{238} \mathrm{U}\end{array}$ & $(2 \sigma)$ & ${ }^{207} \mathrm{~Pb} /{ }^{235} \mathrm{U}$ & $(2 \sigma)$ & ${ }^{207} \mathrm{~Pb} /{ }^{206} \mathrm{~Pb}$ & $(2 \sigma)$ \\
\hline \multicolumn{13}{|l|}{$S A L-12$} \\
\hline ap05a05 & 0.1393 & 0.0006 & 1.3007 & 0.0144 & 0.06772 & 0.00076 & 839 & 7 & 846 & 13 & 860 & 46 \\
\hline ap05a06 & 0.0753 & 0.0003 & 0.5968 & 0.0034 & 0.05745 & 0.0003 & 468 & 4 & 475 & 4 & 508 & 24 \\
\hline ap05a07 & 0.074 & 0.0004 & 0.5933 & 0.0043 & 0.05818 & 0.00048 & 460 & 5 & 473 & 5 & 536 & 36 \\
\hline ap05a08 & 0.2085 & 0.0008 & 2.3722 & 0.0119 & 0.08254 & 0.00043 & 1224 & 9 & 1234 & 7 & 1258 & 20 \\
\hline ap05a09 & 0.0745 & 0.0004 & 0.5829 & 0.0078 & 0.05674 & 0.00073 & 463 & 5 & 466 & 10 & 480 & 56 \\
\hline ap05a10 & 0.0497 & 0.0004 & 0.4598 & 0.0051 & 0.06704 & 0.00052 & 313 & 6 & 384 & 7 & 838 & 32 \\
\hline ap05a11 & 0.2053 & 0.0008 & 2.2962 & 0.017 & 0.08112 & 0.0005 & 1204 & 9 & 1211 & 10 & 1224 & 24 \\
\hline ap05a12 & 0.1712 & 0.0007 & 1.7477 & 0.0147 & 0.07406 & 0.00069 & 1019 & 8 & 1026 & 11 & 1042 & 36 \\
\hline ap05a13 & 0.2104 & 0.0007 & 2.3278 & 0.0242 & 0.08023 & 0.00081 & 1232 & 8 & 1221 & 15 & 1202 & 40 \\
\hline ap05a14 & 0.0737 & 0.0003 & 0.579 & 0.0058 & 0.05695 & 0.00056 & 459 & 4 & 464 & 7 & 488 & 44 \\
\hline ap05a15 & 0.0746 & 0.0003 & 0.5904 & 0.0045 & 0.05744 & 0.0004 & 463 & 4 & 471 & 6 & 508 & 30 \\
\hline ap05a16 & 0.0741 & 0.0003 & 0.6635 & 0.0064 & 0.06495 & 0.00066 & 456 & 3 & 517 & 8 & 772 & 42 \\
\hline ap05b05 & 0.2104 & 0.001 & 2.3768 & 0.0209 & 0.08195 & 0.00074 & 1231 & 11 & 1236 & 13 & 1244 & 34 \\
\hline ap05b06 & 0.2014 & 0.0009 & 2.2188 & 0.0131 & 0.07991 & 0.00051 & 1184 & 11 & 1187 & 8 & 1194 & 24 \\
\hline ap05b07 & 0.0761 & 0.0004 & 0.6075 & 0.0046 & 0.05791 & 0.00043 & 473 & 5 & 482 & 6 & 526 & 34 \\
\hline ap05b08 & 0.1744 & 0.001 & 1.7986 & 0.0214 & 0.0748 & 0.00102 & 1035 & 12 & 1045 & 16 & 1062 & 56 \\
\hline ap05b09 & 0.158 & 0.0008 & 1.5908 & 0.0232 & 0.07301 & 0.00103 & 946 & 9 & 967 & 18 & 1014 & 58 \\
\hline ap05b10 & 0.0751 & 0.0003 & 0.6156 & 0.0057 & 0.05947 & 0.00056 & 466 & 4 & 487 & 7 & 584 & 42 \\
\hline ap05b11 & 0.2019 & 0.001 & 2.2455 & 0.0171 & 0.08068 & 0.00058 & 1186 & 11 & 1195 & 11 & 1212 & 28 \\
\hline ap05b12 & 0.1151 & 0.0008 & 1.0693 & 0.0145 & 0.0674 & 0.00096 & 683 & 11 & 738 & 14 & 850 & 58 \\
\hline ap05b13 & 0.1384 & 0.001 & 1.581 & 0.0207 & 0.08288 & 0.001 & 822 & 12 & 963 & 16 & 1266 & 46 \\
\hline ap05b14 & 0.1368 & 0.0009 & 1.3218 & 0.0169 & 0.07008 & 0.00102 & 822 & 11 & 855 & 15 & 930 & 60 \\
\hline ap05b15 & 0.0759 & 0.0003 & 0.6231 & 0.006 & 0.05958 & 0.00058 & 469 & 3 & 492 & 8 & 588 & 42 \\
\hline ap05b16core & 0.091 & 0.0007 & 0.8237 & 0.0078 & 0.06565 & 0.00069 & 560 & 9 & 610 & 9 & 794 & 44 \\
\hline ap05c05rim & 0.0887 & 0.0003 & 0.808 & 0.0051 & 0.06604 & 0.00045 & 543 & 4 & 601 & 6 & 806 & 28 \\
\hline ap05c06 & 0.0748 & 0.0003 & 0.6124 & 0.0044 & 0.05936 & 0.00047 & 463 & 4 & 485 & 6 & 580 & 34 \\
\hline ap05c07 & 0.0723 & 0.0004 & 0.616 & 0.0056 & 0.06179 & 0.00048 & 448 & 5 & 487 & 7 & 666 & 34 \\
\hline $\begin{array}{l}\text { ap05c08main } \\
\text { zone }\end{array}$ & 0.0694 & 0.0005 & 0.7175 & 0.0067 & 0.075 & 0.00053 & 424 & 7 & 549 & 8 & 1068 & 28 \\
\hline ap05c09rim? & 0.0728 & 0.0002 & 0.5733 & 0.0038 & 0.05715 & 0.00037 & 452 & 3 & 460 & 5 & 496 & 28 \\
\hline ap05c10 & 0.0711 & 0.0003 & 0.9512 & 0.009 & 0.09711 & 0.0009 & 422 & 6 & 679 & 9 & 1568 & 36 \\
\hline ap05c11 & 0.078 & 0.0004 & 0.6415 & 0.0061 & 0.05967 & 0.00061 & 482 & 5 & 503 & 8 & 590 & 44 \\
\hline ap05c12 & 0.0783 & 0.0003 & 0.6511 & 0.0077 & 0.0603 & 0.00072 & 486 & 4 & 509 & 9 & 614 & 50 \\
\hline ap $05 \mathrm{c} 13$ & 0.1697 & 0.0008 & 1.8473 & 0.0168 & 0.07896 & 0.00076 & 1001 & 9 & 1062 & 12 & 1170 & 36 \\
\hline ap $05 \mathrm{c} 14$ core & 0.0726 & 0.0004 & 0.5647 & 0.0067 & 0.05639 & 0.00061 & 452 & 6 & 455 & 9 & 466 & 48 \\
\hline ap05c15rim & 0.0735 & 0.0004 & 0.5659 & 0.008 & 0.05582 & 0.00078 & 457 & 5 & 455 & 10 & 444 & 60 \\
\hline ap05c16 & 0.1299 & 0.0011 & 1.287 & 0.0257 & 0.07189 & 0.00129 & 765 & 15 & 840 & 23 & 982 & 74 \\
\hline ap05d05 & 0.1661 & 0.0005 & 1.6569 & 0.0101 & 0.07236 & 0.00045 & 991 & 6 & 992 & 8 & 994 & 26 \\
\hline ap05d06 & 0.0743 & 0.0003 & 0.5738 & 0.006 & 0.05603 & 0.00054 & 463 & 4 & 460 & 8 & 452 & 44 \\
\hline ap05d07 & 0.1822 & 0.0011 & 1.9441 & 0.0247 & 0.07741 & 0.00098 & 1076 & 13 & 1096 & 17 & 1130 & 50 \\
\hline ap05d08 & 0.0721 & 0.0003 & 0.6582 & 0.0047 & 0.06622 & 0.00045 & 445 & 4 & 514 & 6 & 812 & 28 \\
\hline ap05d09 & 0.1518 & 0.0008 & 1.4925 & 0.0161 & 0.07132 & 0.00081 & 912 & 9 & 927 & 13 & 966 & 46 \\
\hline ap05d10 & 0.0737 & 0.0003 & 0.7233 & 0.0073 & 0.0712 & 0.00077 & 451 & 4 & 553 & 9 & 962 & 44 \\
\hline ap05d11 & 0.2747 & 0.0012 & 3.7626 & 0.026 & 0.09936 & 0.00062 & 1565 & 12 & 1585 & 11 & 1612 & 22 \\
\hline ap05d12 & 0.2208 & 0.0012 & 2.606 & 0.0305 & 0.08561 & 0.00104 & 1280 & 14 & 1302 & 17 & 1328 & 48 \\
\hline ap $05 \mathrm{~d} 13$ & 0.0747 & 0.0003 & 0.5832 & 0.0045 & 0.05662 & 0.0004 & 465 & 4 & 467 & 6 & 476 & 32 \\
\hline ap05d14 & 0.0746 & 0.0004 & 0.586 & 0.0064 & 0.05697 & 0.00063 & 463 & 5 & 468 & 8 & 490 & 48 \\
\hline ap05d15 & 0.1821 & 0.001 & 2.0148 & 0.025 & 0.08025 & 0.00101 & 1076 & 12 & 1121 & 17 & 1202 & 48 \\
\hline ap $05 \mathrm{~d} 16$ & 0.0743 & 0.0004 & 0.5703 & 0.0094 & 0.05567 & 0.00097 & 463 & 5 & 458 & 12 & 438 & 76 \\
\hline ap05e05 & 0.0764 & 0.0003 & 0.7428 & 0.0065 & 0.07055 & 0.00068 & 467 & 4 & 564 & 8 & 944 & 38 \\
\hline ap05e06 & 0.0741 & 0.0004 & 0.5804 & 0.0059 & 0.05677 & 0.00052 & 461 & 5 & 465 & 8 & 482 & 40 \\
\hline ap05e07 & 0.149 & 0.0004 & 1.4563 & 0.0063 & 0.0709 & 0.00026 & 895 & 5 & 912 & 5 & 954 & 16 \\
\hline ap05e08 & 0.1832 & 0.0008 & 2.2745 & 0.015 & 0.09004 & 0.0005 & 1077 & 9 & 1204 & 9 & 1426 & 22 \\
\hline ap05e09 & 0.0735 & 0.0003 & 0.8316 & 0.0063 & 0.08209 & 0.00065 & 444 & 5 & 615 & 7 & 1246 & 30 \\
\hline
\end{tabular}

\subsection{Jurassic conglomerate}

Lying unconformably upon the Tecomate Formation in the northeastern part of the area are a sequence of red conglomer- ates, sandstones and occasional shaly layers (Fig. 3). Leaf impressions recovered from this sequence are probably Jurassic (R. Weber, pers. comm., 2005). The conglomerates contain pebbles of schist and large feldspar and quartz grains. These 
Table 5

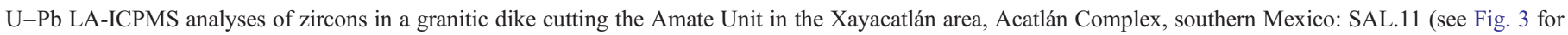
sample locations)

\begin{tabular}{|c|c|c|c|c|c|c|c|c|c|c|c|c|}
\hline Sample/analysis & ${ }^{206} \mathrm{~Pb} /{ }^{238} \mathrm{U}$ & $\begin{array}{l}\text { Percentage } \\
\text { error }\end{array}$ & ${ }^{207} \mathrm{~Pb} /{ }^{235} \mathrm{U}$ & $\begin{array}{l}\text { Percentage } \\
\text { error }\end{array}$ & ${ }^{207} \mathrm{~Pb} /{ }^{206} \mathrm{~Pb}$ & $\begin{array}{l}\text { Percentage } \\
\text { error }\end{array}$ & $\begin{array}{l}\text { Age, Ma } \\
{ }^{206} \mathrm{~Pb} /{ }^{238} \mathrm{U}\end{array}$ & $(2 \sigma)$ & ${ }^{207} \mathrm{~Pb} /{ }^{235} \mathrm{U}$ & $(2 \sigma)$ & ${ }^{207} \mathrm{~Pb} /{ }^{206} \mathrm{~Pb}$ & $(2 \sigma)$ \\
\hline \multicolumn{13}{|l|}{$S A L-11$} \\
\hline ap03a05 & 0.5925 & 0.0023 & 19.7945 & 0.0673 & 0.24231 & 0.00073 & 3000 & 19 & 3081 & 7 & 3134 & 8 \\
\hline ap03a06 & 0.1097 & 0.0005 & 0.9668 & 0.0079 & 0.0639 & 0.00048 & 671 & 6 & 687 & 8 & 738 & 32 \\
\hline ap03a07 & 0.1689 & 0.0006 & 1.7389 & 0.0144 & 0.07468 & 0.00063 & 1008 & 7 & 1023 & 11 & 1058 & 34 \\
\hline ap03a08 & 0.1997 & 0.0013 & 2.3 & 0.0455 & 0.08352 & 0.00159 & 1174 & 16 & 1212 & 28 & 1280 & 74 \\
\hline ap03a09 & 0.5024 & 0.0022 & 11.8614 & 0.0474 & 0.17123 & 0.00067 & 2628 & 19 & 2594 & 8 & 2568 & 14 \\
\hline ap03a10 & 0.098 & 0.0005 & 0.8209 & 0.0069 & 0.06076 & 0.00058 & 602 & 6 & 609 & 8 & 630 & 42 \\
\hline ap03a11 & 0.0871 & 0.0005 & 0.7168 & 0.0116 & 0.05967 & 0.00092 & 538 & 6 & 549 & 14 & 590 & 68 \\
\hline ap03a12 & 0.1045 & 0.0005 & 0.8922 & 0.0062 & 0.06194 & 0.00046 & 641 & 5 & 648 & 7 & 670 & 32 \\
\hline ap03a13 & 0.3512 & 0.0012 & 12.1767 & 0.0426 & 0.25151 & 0.0007 & 1940 & 11 & 2618 & 7 & 3194 & 10 \\
\hline ap03a14 & 0.2776 & 0.0012 & 4.1555 & 0.0258 & 0.10858 & 0.00073 & 1566 & 13 & 1665 & 10 & 1774 & 26 \\
\hline ap03a15 & 0.0851 & 0.0006 & 0.7059 & 0.0077 & 0.06015 & 0.00067 & 527 & 8 & 542 & 9 & 608 & 48 \\
\hline ap03a16 & 0.0973 & 0.0005 & 0.7968 & 0.01 & 0.05942 & 0.00078 & 599 & 6 & 595 & 11 & 582 & 58 \\
\hline ap03b05 & 0.134 & 0.0007 & 1.2375 & 0.0099 & 0.06697 & 0.00051 & 812 & 8 & 818 & 9 & 836 & 32 \\
\hline ap03b06 & 0.3057 & 0.002 & 5.1402 & 0.0334 & 0.12198 & 0.00059 & 1717 & 21 & 1843 & 11 & 1984 & 16 \\
\hline ap03b07 & 0.3356 & 0.0012 & 5.2714 & 0.0285 & 0.11394 & 0.00062 & 1869 & 13 & 1864 & 9 & 1862 & 20 \\
\hline ap03b08 & 0.1777 & 0.0009 & 1.8322 & 0.0114 & 0.07479 & 0.0004 & 1055 & 11 & 1057 & 8 & 1062 & 20 \\
\hline ap03b09 & 0.2049 & 0.0008 & 2.3866 & 0.0155 & 0.0845 & 0.00046 & 1198 & 8 & 1239 & 9 & 1304 & 22 \\
\hline ap03b10 & 0.14 & 0.0006 & 1.3963 & 0.0101 & 0.07235 & 0.00046 & 839 & 7 & 887 & 8 & 994 & 26 \\
\hline ap03b11 & 0.1022 & 0.0004 & 0.8567 & 0.0072 & 0.06078 & 0.00051 & 629 & 5 & 628 & 8 & 630 & 36 \\
\hline ap03b12 & 0.2012 & 0.0008 & 2.1772 & 0.0155 & 0.0785 & 0.00057 & 1184 & 10 & 1174 & 10 & 1158 & 28 \\
\hline ap03b13 & 0.1025 & 0.0006 & 0.9125 & 0.0115 & 0.06455 & 0.00077 & 627 & 7 & 658 & 12 & 758 & 50 \\
\hline ap03b14 & 0.302 & 0.0009 & 4.4817 & 0.0134 & 0.10763 & 0.00042 & 1699 & 10 & 1728 & 5 & 1758 & 14 \\
\hline ap03b15 & 0.0829 & 0.0007 & 1.2626 & 0.0758 & 0.11048 & 0.00514 & 483 & 14 & 829 & 68 & 1806 & 168 \\
\hline ap03b16 & 0.2202 & 0.0084 & 16.4658 & 0.7014 & 0.54224 & 0.00672 & 492 & 220 & 2904 & 82 & 4000 & 0 \\
\hline ap03c05 & 0.1496 & 0.0047 & 2.9584 & 0.0846 & 0.14347 & 0.00194 & 847 & 60 & 1397 & 43 & 2268 & 46 \\
\hline ap03c06 & 0.1 & 0.0008 & 1.1146 & 0.0308 & 0.08087 & 0.00215 & 604 & 12 & 760 & 30 & 1218 & 104 \\
\hline ap03c07 & 0.1533 & 0.0007 & 1.493 & 0.0094 & 0.07063 & 0.00037 & 919 & 8 & 928 & 8 & 946 & 20 \\
\hline ap03c08 & 0.0724 & 0.0003 & 0.5811 & 0.0035 & 0.05823 & 0.00037 & 450 & 4 & 465 & 4 & 538 & 28 \\
\hline ap03c09 & 0.0617 & 0.0003 & 0.6057 & 0.0072 & 0.07117 & 0.00088 & 380 & 5 & 481 & 9 & 962 & 50 \\
\hline ap03c10 & 0.1779 & 0.0007 & 1.8184 & 0.0156 & 0.07413 & 0.00066 & 1056 & 9 & 1052 & 11 & 1044 & 36 \\
\hline ap03c11 & 0.0872 & 0.0004 & 0.7705 & 0.0065 & 0.06408 & 0.00062 & 544 & 5 & 580 & 7 & 744 & 42 \\
\hline ap03c12 & 0.3317 & 0.001 & 5.1702 & 0.0248 & 0.11305 & 0.00047 & 1848 & 10 & 1848 & 8 & 1848 & 16 \\
\hline ap03c13 & 0.1044 & 0.0005 & 0.991 & 0.0076 & 0.06884 & 0.00049 & 639 & 6 & 699 & 8 & 892 & 30 \\
\hline ap03c14 & 0.1481 & 0.0015 & 1.9885 & 0.0169 & 0.09736 & 0.00152 & 875 & 18 & 1112 & 11 & 1574 & 58 \\
\hline ap03c15 & 0.149 & 0.0006 & 1.7949 & 0.0179 & 0.0874 & 0.00087 & 876 & 8 & 1044 & 13 & 1368 & 38 \\
\hline ap03c16 & 0.065 & 0.0003 & 0.7303 & 0.0067 & 0.08152 & 0.00088 & 395 & 5 & 557 & 8 & 1232 & 42 \\
\hline ap03d05 & 0.0753 & 0.0002 & 0.5968 & 0.0054 & 0.05748 & 0.00056 & 469 & 3 & 475 & 7 & 508 & 42 \\
\hline ap03d06 & 0.0685 & 0.0004 & 0.5218 & 0.0078 & 0.05524 & 0.0008 & 434 & 7 & 426 & 10 & 422 & 66 \\
\hline ap03d07 & 0.0842 & 0.0004 & 0.6861 & 0.0096 & 0.05913 & 0.00079 & 521 & 6 & 530 & 12 & 570 & 58 \\
\hline ap03d08 & 0.1016 & 0.0005 & 0.8589 & 0.0071 & 0.06134 & 0.00059 & 624 & 7 & 630 & 8 & 650 & 40 \\
\hline ap03d09 & 0.1009 & 0.0005 & 0.8772 & 0.0113 & 0.06304 & 0.00093 & 620 & 6 & 639 & 12 & 708 & 64 \\
\hline ap03d10 & 0.5241 & 0.0023 & 13.6093 & 0.049 & 0.18836 & 0.00068 & 2710 & 21 & 2723 & 7 & 2726 & 12 \\
\hline ap03d11 & 0.0971 & 0.0006 & 0.8556 & 0.0076 & 0.06392 & 0.00062 & 597 & 7 & 628 & 8 & 738 & 40 \\
\hline ap03d12 & 0.1221 & 0.0004 & 1.0863 & 0.0086 & 0.06451 & 0.00049 & 742 & 5 & 747 & 8 & 758 & 32 \\
\hline ap03d13 & 0.1032 & 0.0007 & 0.9907 & 0.0141 & 0.06964 & 0.0011 & 626 & 9 & 699 & 14 & 916 & 66 \\
\hline ap03d14 & 0.1008 & 0.0004 & 0.8459 & 0.0087 & 0.06089 & 0.00062 & 621 & 5 & 622 & 10 & 634 & 44 \\
\hline ap03d15 & 0.0534 & 0.0003 & 0.4393 & 0.0066 & 0.05968 & 0.00089 & 334 & 4 & 370 & 9 & 592 & 66 \\
\hline ap03d16 & 0.0832 & 0.0012 & 0.9478 & 0.0181 & 0.08259 & 0.00249 & 500 & 16 & 677 & 19 & 1258 & 118 \\
\hline ap03e05 & 0.1246 & 0.0007 & 1.1595 & 0.0256 & 0.06749 & 0.00115 & 754 & 9 & 782 & 24 & 852 & 72 \\
\hline ap03e06 & 0.1456 & 0.0006 & 1.8269 & 0.011 & 0.09099 & 0.00056 & 885 & 8 & 1055 & 8 & 1446 & 24 \\
\hline ap03e07 & 0.1905 & 0.001 & 2.1608 & 0.0212 & 0.08227 & 0.00079 & 1122 & 12 & 1169 & 14 & 1250 & 38 \\
\hline ap03e08 & 0.0872 & 0.0004 & 0.7177 & 0.0093 & 0.05967 & 0.00083 & 539 & 5 & 549 & 11 & 590 & 60 \\
\hline ap03e09 & 0.3321 & 0.0024 & 8.2825 & 0.0654 & 0.1809 & 0.00166 & 1822 & 28 & 2262 & 14 & 2660 & 32 \\
\hline ap03e10 & 0.0824 & 0.0004 & 0.6537 & 0.0093 & 0.05755 & 0.00079 & 511 & 6 & 511 & 11 & 512 & 62 \\
\hline ap03e11 & 0.2037 & 0.0008 & 2.2611 & 0.0154 & 0.0805 & 0.00049 & 1195 & 10 & 1200 & 10 & 1208 & 24 \\
\hline ap03e12 & 0.056 & 0.0002 & 0.4169 & 0.0042 & 0.05398 & 0.00062 & 352 & 3 & 354 & 6 & 370 & 52 \\
\hline ap03e13 & 0.1016 & 0.0004 & 0.8675 & 0.0107 & 0.06192 & 0.00074 & 623 & 6 & 634 & 12 & 670 & 52 \\
\hline ap03e14 & 0.1165 & 0.0005 & 1.0567 & 0.0123 & 0.06581 & 0.00083 & 709 & 7 & 732 & 12 & 800 & 54 \\
\hline ap03e15 & 0.0736 & 0.0004 & 0.5859 & 0.0057 & 0.05772 & 0.00055 & 459 & 5 & 468 & 7 & 518 & 42 \\
\hline ap03e16 & 0.0783 & 0.0004 & 0.6278 & 0.007 & 0.05819 & 0.00066 & 485 & 5 & 495 & 9 & 536 & 50 \\
\hline
\end{tabular}


Table 5 (continued)

\begin{tabular}{|c|c|c|c|c|c|c|c|c|c|c|c|c|}
\hline Sample/analysis & ${ }^{206} \mathrm{~Pb} /{ }^{238} \mathrm{U}$ & $\begin{array}{l}\text { Percentage } \\
\text { error }\end{array}$ & ${ }^{207} \mathrm{~Pb} /{ }^{235} \mathrm{U}$ & $\begin{array}{l}\text { Percentage } \\
\text { error }\end{array}$ & ${ }^{207} \mathrm{~Pb} /{ }^{206} \mathrm{~Pb}$ & $\begin{array}{l}\text { Percentage } \\
\text { error }\end{array}$ & $\begin{array}{l}\text { Age, Ma } \\
{ }^{206} \mathrm{~Pb} /{ }^{238} \mathrm{U}\end{array}$ & $(2 \sigma)$ & ${ }^{207} \mathrm{~Pb} /{ }^{235} \mathrm{U}$ & $(2 \sigma)$ & ${ }^{207} \mathrm{~Pb} /{ }^{206} \mathrm{~Pb}$ & $(2 \sigma)$ \\
\hline \multicolumn{13}{|l|}{$S A L-11$} \\
\hline ap03f05 & 0.1581 & 0.0006 & 1.9772 & 0.0091 & 0.09068 & 0.00052 & 918 & 9 & 1108 & 6 & 1438 & 22 \\
\hline ap03f06 & 0.1646 & 0.0011 & 1.7427 & 0.0197 & 0.07677 & 0.0009 & 973 & 14 & 1024 & 15 & 1114 & 48 \\
\hline ap03f07 & 0.1534 & 0.0013 & 1.5557 & 0.0219 & 0.07355 & 0.00088 & 918 & 16 & 953 & 17 & 1028 & 48 \\
\hline ap03f08 & 0.1578 & 0.0006 & 1.6096 & 0.0106 & 0.074 & 0.00046 & 943 & 7 & 974 & 8 & 1040 & 26 \\
\hline ap03f09 & 0.1332 & 0.0012 & 1.2409 & 0.045 & 0.06758 & 0.00219 & 801 & 16 & 819 & 41 & 854 & 134 \\
\hline ap03f10 & 0.0992 & 0.0006 & 0.8582 & 0.0094 & 0.06278 & 0.00082 & 608 & 7 & 629 & 10 & 700 & 56 \\
\hline ap03f11 & 0.1048 & 0.0008 & 0.9372 & 0.0378 & 0.06487 & 0.00288 & 640 & 11 & 671 & 40 & 770 & 188 \\
\hline ap03f12 & 0.3579 & 0.0017 & 5.9463 & 0.0416 & 0.1205 & 0.00081 & 1974 & 19 & 1968 & 12 & 1962 & 24 \\
\hline ap03f13 & 0.203 & 0.0009 & 2.337 & 0.0236 & 0.08352 & 0.00071 & 1190 & 10 & 1224 & 14 & 1280 & 32 \\
\hline ap03f14 & 0.3538 & 0.0029 & 7.712 & 0.0671 & 0.15812 & 0.00059 & 1936 & 31 & 2198 & 16 & 2434 & 12 \\
\hline ap03f15 & 0.1801 & 0.0009 & 1.903 & 0.0124 & 0.07662 & 0.00056 & 1065 & 10 & 1082 & 9 & 1110 & 28 \\
\hline ap03f16 & 0.0887 & 0.0009 & 0.7442 & 0.0258 & 0.06085 & 0.0022 & 546 & 11 & 565 & 30 & 632 & 156 \\
\hline ap03g05 & 0.0908 & 0.0003 & 0.8468 & 0.007 & 0.06768 & 0.00057 & 554 & 5 & 623 & 8 & 858 & 34 \\
\hline ap03g06 & 0.0868 & 0.0006 & 0.7051 & 0.0113 & 0.05893 & 0.00098 & 536 & 7 & 542 & 13 & 564 & 74 \\
\hline ap03g07 & 0.0917 & 0.0005 & 0.7766 & 0.0077 & 0.06142 & 0.00073 & 566 & 6 & 584 & 9 & 652 & 50 \\
\hline ap03g08 & 0.0572 & 0.0002 & 0.4342 & 0.0066 & 0.05508 & 0.00089 & 358 & 3 & 366 & 9 & 414 & 72 \\
\hline ap03g09 & 0.0564 & 0.0002 & 0.4184 & 0.0033 & 0.05377 & 0.00042 & 352 & 3 & 355 & 5 & 360 & 36 \\
\hline ap03g10 & 0.0766 & 0.0004 & 0.6183 & 0.0098 & 0.05852 & 0.00105 & 476 & 6 & 489 & 12 & 548 & 80 \\
\hline ap03g11 & 0.0966 & 0.0005 & 0.8187 & 0.009 & 0.06146 & 0.00061 & 593 & 6 & 607 & 10 & 654 & 44 \\
\hline ap03g12 & 0.1022 & 0.0004 & 0.8782 & 0.0056 & 0.06234 & 0.00043 & 627 & 5 & 640 & 6 & 684 & 30 \\
\hline ap03g13 & 0.1485 & 0.0009 & 1.4725 & 0.0141 & 0.0719 & 0.00079 & 889 & 11 & 919 & 12 & 982 & 44 \\
\hline ap03g14 & 0.0916 & 0.0004 & 0.8903 & 0.0112 & 0.07052 & 0.00089 & 559 & 6 & 647 & 12 & 942 & 52 \\
\hline ap03g15 & 0.1826 & 0.0005 & 1.9295 & 0.0125 & 0.07664 & 0.00055 & 1082 & 6 & 1091 & 9 & 1110 & 30 \\
\hline ap03g16 & 0.3392 & 0.0013 & 5.4985 & 0.0302 & 0.11758 & 0.00061 & 1882 & 14 & 1900 & 9 & 1918 & 18 \\
\hline
\end{tabular}

rocks are gently dipping to the north and post-date all but normal faulting.

\section{LA-ICPMS zircon analyses}

\subsection{Analytical procedures}

Preparation of the five samples was carried at Instituto de Geología, UNAM. About $15 \mathrm{~kg}$ of each of the selected samples for zircon dating was crushed with a jaw crusher and pulverized in a Bico disk mill. The heavy minerals were concentrated on a Wifley table and were then passed through a Frantz magnetic separator. A current up to $1.5 \mathrm{~A}$ is used to generate a magnetic field for samples to concentrate zircons suitable for LA-ICPMS dating. Further separation by passing non-magnetic fractions through heavy liquids (bromoform), ensured a nearly pure zircon concentration.

Zircons from all five samples were mounted in epoxy and polished for age determinations on individual grains by LAICPMS. All grains were photographed using transmitted and reflected light optical microscopy, and the internal structures of the grains imaged using SEM-CL. U-Pb isotopic compositions of individual zircon grains were determined at RSES-ANU, Canberra, Australia, using an ArF excimer laser system (193 nm, Lambda Physik) coupled to an Agilent 7500 quadrupole ICPMS (Eggins et al., 1998). Ablation was done under a He atmosphere in a custom-built sample chamber using a 40-micron diameter spot and a laser repetition rate of $4 \mathrm{~Hz}$. Data were reduced relative to the reference zircon FC1 (1099 Ma, Paces and Miller 1993), with gas backgrounds collected before each analysis and subtracted from net intensities measured for each isotope. Internal precision of measured ${ }^{207} \mathrm{~Pb} /{ }^{206} \mathrm{~Pb}$ and ${ }^{206} \mathrm{~Pb} /{ }^{238} \mathrm{U}$ ratios typically was $\sim 0.5 \%$ $1 \sigma$ relative standard deviation for individual analyses. The samples were analyzed in two sessions; replicate analyses of the FC1 zircon from each session indicate external reproducibilities of $0.9 \%$ $(n=30)$ and $0.8 \%(n=32)$, respectively, on the measured ${ }^{207} \mathrm{~Pb} /{ }^{206} \mathrm{~Pb}$ ratios, $1.1 \%$ and $0.8 \%$, respectively, on the measured ${ }^{206} \mathrm{~Pb} /{ }^{238} \mathrm{U}$ ratios, and $1.2 \%$ and $1.6 \%$, respectively, on the ${ }^{208} \mathrm{~Pb} /{ }^{232} \mathrm{Th}$ ratios ( $\sigma$ relative standard deviation). These errors are not included in the quoted uncertainties for individual analyses of the analyzed zircons. The data are provided in Tables $1-5$ and the corrected ${ }^{206} \mathrm{~Pb} /{ }^{238} \mathrm{U}$ data are plotted on Terra-Wasserburg diagrams (Figs. 4-8).

\section{Results}

A pegmatite cutting the eastern part of the Huerta Unit (HUERTA-1) consists of K-feldspar, quartz and muscovite with accessory zircon, apatite and opaque minerals. Zircons yielded ${ }^{206} \mathrm{~Pb} /{ }^{238} \mathrm{U}$ ages ranging from $427 \mathrm{Ma}$ to $2531 \mathrm{Ma}$, however the youngest, almost concordant ${ }^{206} \mathrm{~Pb} /{ }^{238} \mathrm{U}$ zircon age is $464 \pm 4 \mathrm{Ma}$ and a mean age of the four almost concordant young zircons is $\sim 485.5 \mathrm{Ma}$ (Fig. 4; Table 1): these are interpreted as dating the time of intrusion. Two population of older, inherited zircons with concordant ages have ages of $\sim 530-580 \mathrm{Ma}$ and $\sim 910-1200 \mathrm{Ma}$.

Three samples were collected from the Amate Unit: one feldspathic metapsammite (AMATE-1) and two cross-cutting, foliated granitic dikes (AMATE-2 and SAL-12). The metapsammite is composed mainly of quartz, K-feldspar and muscovite. It yielded detrital zircons with ${ }^{206} \mathrm{~Pb} /{ }^{238} \mathrm{U}$ ages ranging from $847 \mathrm{Ma}$ to $1325 \mathrm{Ma}$, however, the youngest concordant detrital zircon gave 


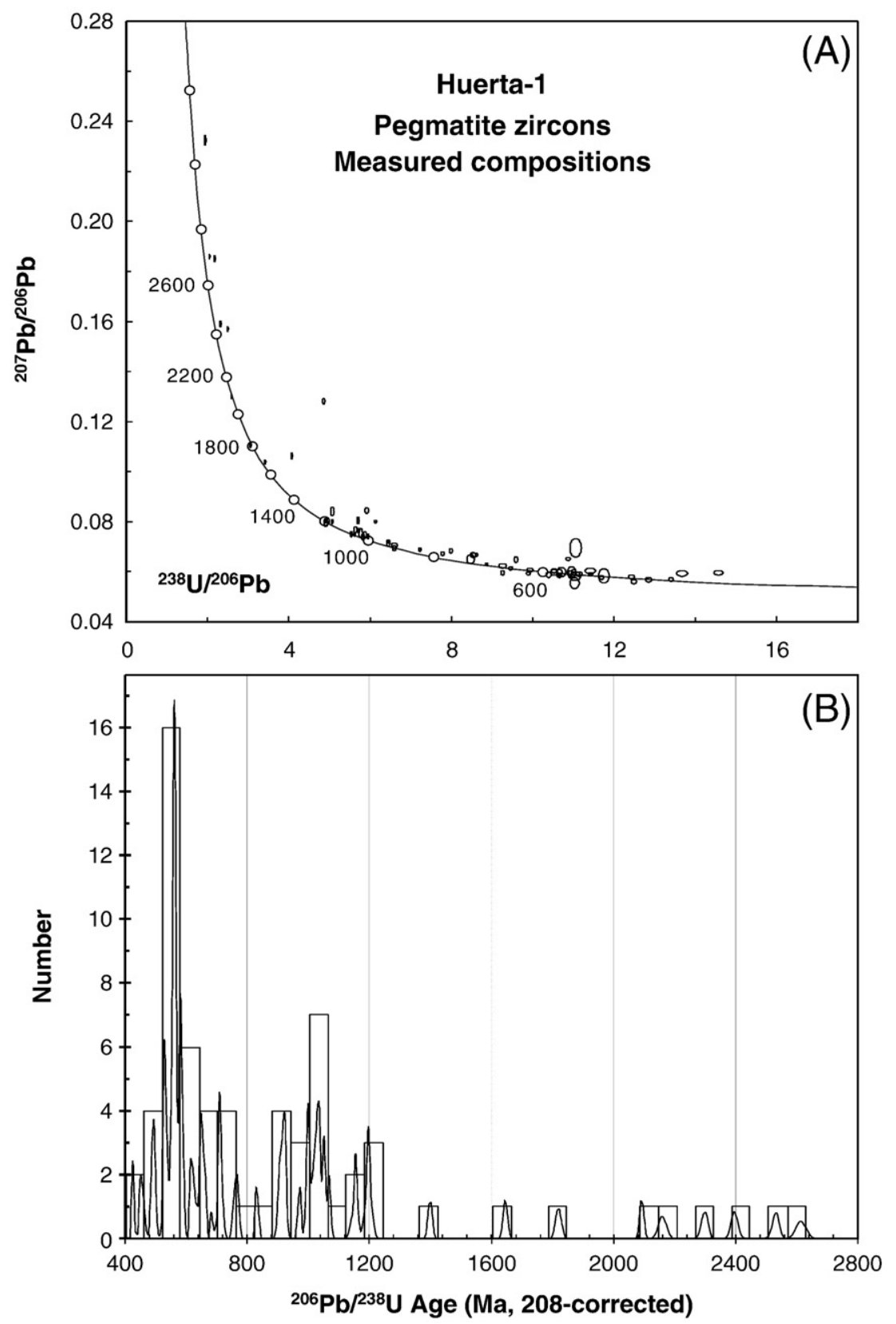

Fig. 4. U-Pb LA-ICPMS zircon analyses from pegmatite cutting the Huerta Unit (sample HUERTA-1) plotted on (A) a Terra-Wasserburg diagram, and (B) a histogram.

$\mathrm{a}{ }^{206} \mathrm{~Pb} /{ }^{238} \mathrm{U}$ age of $902 \pm 14 \mathrm{Ma}$, and older concordant ages in the range of $\sim 900 \mathrm{Ma}$ to $1300 \mathrm{Ma}$ (Fig. 5: Table 2).

The foliated granite dike (AMATE-2) consists of quartz, feldspar, muscovite, and accessory zircon, apatite, and opaque minerals. The ${ }^{206} \mathrm{~Pb} /{ }^{238} \mathrm{U}$ ages range from $432 \mathrm{Ma}$ to $1252 \mathrm{Ma}$ with the youngest concordant ${ }^{206} \mathrm{~Pb} /{ }^{238} \mathrm{U}$ zircon age being $447 \pm$ $3 \mathrm{Ma}$ (Fig. 6, Table 3). Older inherited zircon ages with concordant ${ }^{206} \mathrm{~Pb} /{ }^{238} \mathrm{U}$ ages in the range of 950-1230 Ma.

Another foliated and folded granitic dike (SAL-12) intruding the eastern part of the Amate Unit yielded ${ }^{206} \mathrm{~Pb} /{ }^{238} \mathrm{U}$ zircon ages ranging from $422 \mathrm{Ma}$ to $1565 \mathrm{Ma}$, however the youngest concordant age is $452 \pm 6 \mathrm{Ma}$ interpreted as the time of intrusion (Fig. 7, Table 4). Older inherited zircons gave concordant ${ }^{206} \mathrm{~Pb} /{ }^{238} \mathrm{U}$ ages in the range of $912-1280 \mathrm{Ma}$ with single concordant ages at $839 \pm 7 \mathrm{Ma}$ and $1565 \pm 12 \mathrm{Ma}$.
Detrital zircons in a metapsammite of the Salada Unit (SAL-11) yielded a ${ }^{206} \mathrm{~Pb} /{ }^{238} \mathrm{U}$ ranging from $352 \mathrm{Ma}$ to $3000 \mathrm{Ma}$ with the youngest concordant detrital zircon yielded a ${ }^{206} \mathrm{~Pb} /{ }^{238} \mathrm{U}$ age of $352 \pm 3 \mathrm{Ma}$ (Fig. 8, Table 5). Older concordant ages of -434-485 Ma, 511-630 Ma, and 920-1200 Ma.

\section{Constraints on the ages of the new units}

\subsection{Huerta Unit}

Deposition of the Huerta Unit is constrained by the $455 \pm 4 \mathrm{Ma}$ age of the youngest detrital zircon (Keppie et al., 2006) and 464 \pm $4 \mathrm{Ma}$ intrusive age of a cross-cutting pegmatite. The ages are similar within analytical error and suggest that deposition was synchronous with intrusion. However, the two dated samples were collected 

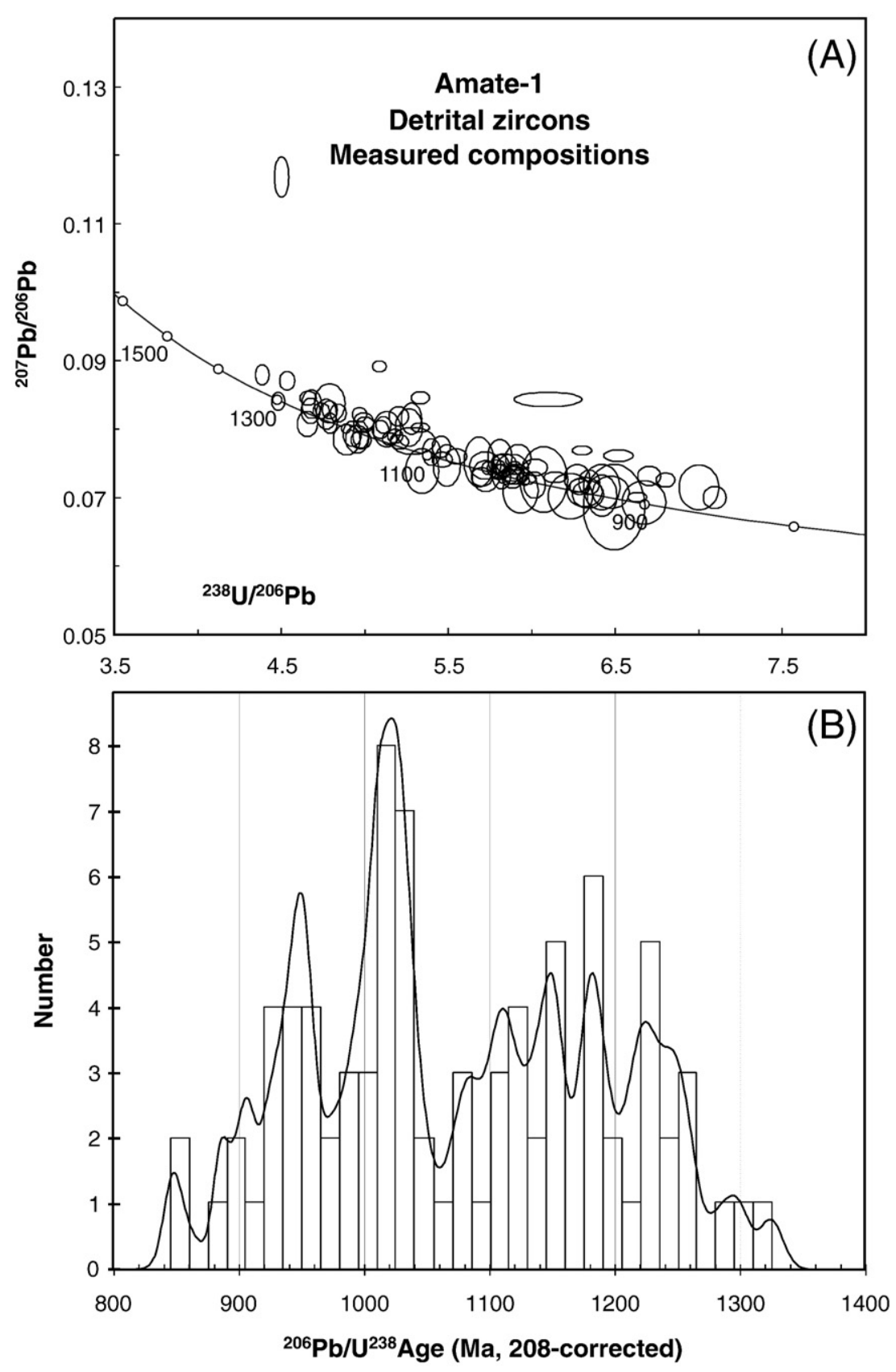

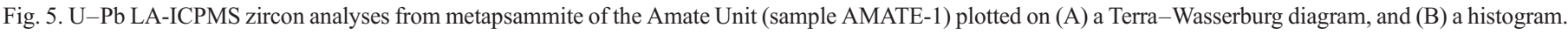

$10 \mathrm{~km}$ apart from western and eastern parts of the Huerta Unit, respectively. Thus deposition of rocks in the eastern part of the unit that are cut by the pegmatite may be somewhat older than $\sim 455 \mathrm{Ma}$. The polyphase deformation of the unit probably means that this problem cannot be ascertained by further mapping: further detrital zircon dating is required. Nevertheless, deposition of the unit probably took place during the Ordovician.

\subsection{Amate Unit}

Deposition of the Amate Unit is less tightly constrained: post-902 $\pm 14 \mathrm{Ma}$ (youngest concordant detrital zircon) and pre$447 \pm 3 \mathrm{Ma}$, pre- $452 \pm 6 \mathrm{Ma}$ (the intrusive ages of cross-cutting granite dikes: this paper), and pre- $442 \pm 1 \mathrm{Ma}$ (the intrusive age of a cross-cutting gabbro dike: Keppie et al., 2008b). The absence of late Neoproterozoic ages in the Amate Unit, where they are present in the Huerta Unit, suggests either that the units are of different ages or that the provenance changed. The latter situation is apparent in the Ordovician units of the Patlanoaya area (Keppie et al., 2008b) and in the Tecomate Formation (Sánchez-Zavala et al., 2004). However, at this stage, one cannot rule out a difference in the time of deposition.

\subsection{Salada Unit}

An older limit on the time of deposition of the Salada Unit is provided by the $352 \pm 3 \mathrm{Ma}$ age of the youngest detrital zircon. A younger limit is not directly available. The Tecomate 

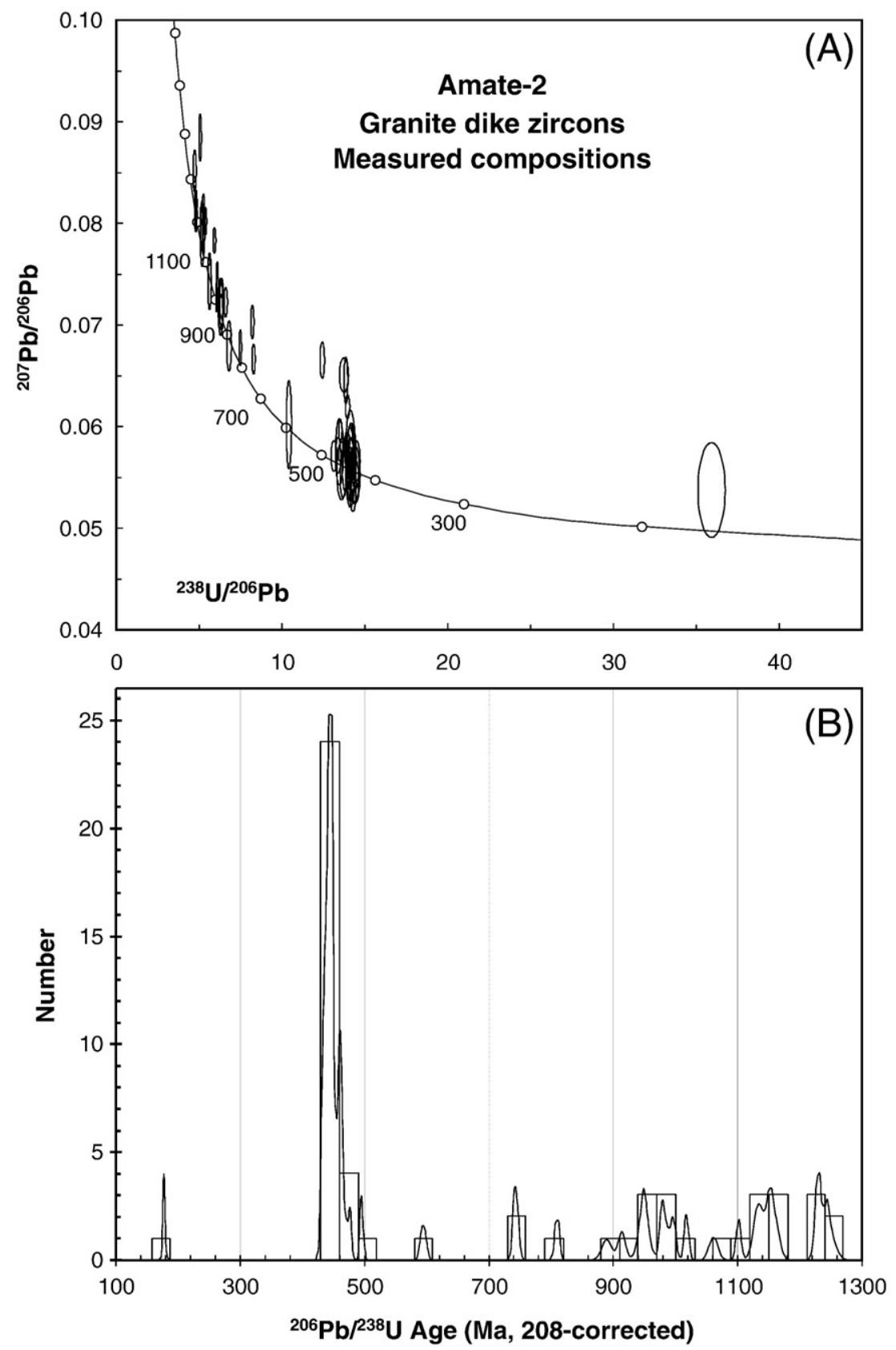

Fig. 6. U-Pb LA-ICPMS zircon analyses from granitic dike cutting the Amate Unit (sample AMATE-2) plotted on (A) a Terra-Wasserburg diagram, and (B) a histogram.

Formation is everywhere in tectonic contact with the Salada Unit, however, the lithologies are markedly distinct and the dated parts of the Tecomate Formation are latest Pennsylvanian to Middle Permian (Keppie et al., 2004a). Furthermore, it is lithologically distinct from the Permo-Triassic Chazumba and Magdalena units, which also have distinctly younger detrital zircon populations (Keppie et al., 2006). Thus its age is most likely to be Carboniferous.

\section{Provenance of the detrital zircons}

Whereas both the Huerta and Amate units both contain a 900-1300 Ma detrital zircon population, $\sim 460 \mathrm{Ma}$ and $\sim 510$
640 Ma populations are only recorded in the Huerta Unit. The 900-1300 Ma population is probably of local provenance in the neighbouring Oaxacan Complex (Fig. 1B), which has yielded ages ranging from $920 \mathrm{Ma}$ to $1300 \mathrm{Ma}$ (Keppie et al., 2003; Solari et al., 2003; Ortega-Obregón et al., 2003). A local source for the $\sim 460 \mathrm{Ma}$ detrital zircons is also likely in the Ordovician granitoids of the Acatlán Complex (Fig. 1B)(Sánchez-Zavala et al., 2004; Talavera-Mendoza et al., 2005; Miller et al., 2007). On the other hand a more distal source for the $\sim 510-640 \mathrm{Ma}$ zircons may be found in either the basement beneath the Yucatan Peninsula, the Brasiliano orogens of Amazonia, or Avalonia (which separated from Oaxaquia/Amazonia at $\sim 480 \mathrm{Ma}$ ) (Keppie, 2004; Keppie et al., 2006; Nance et al., 2006). 


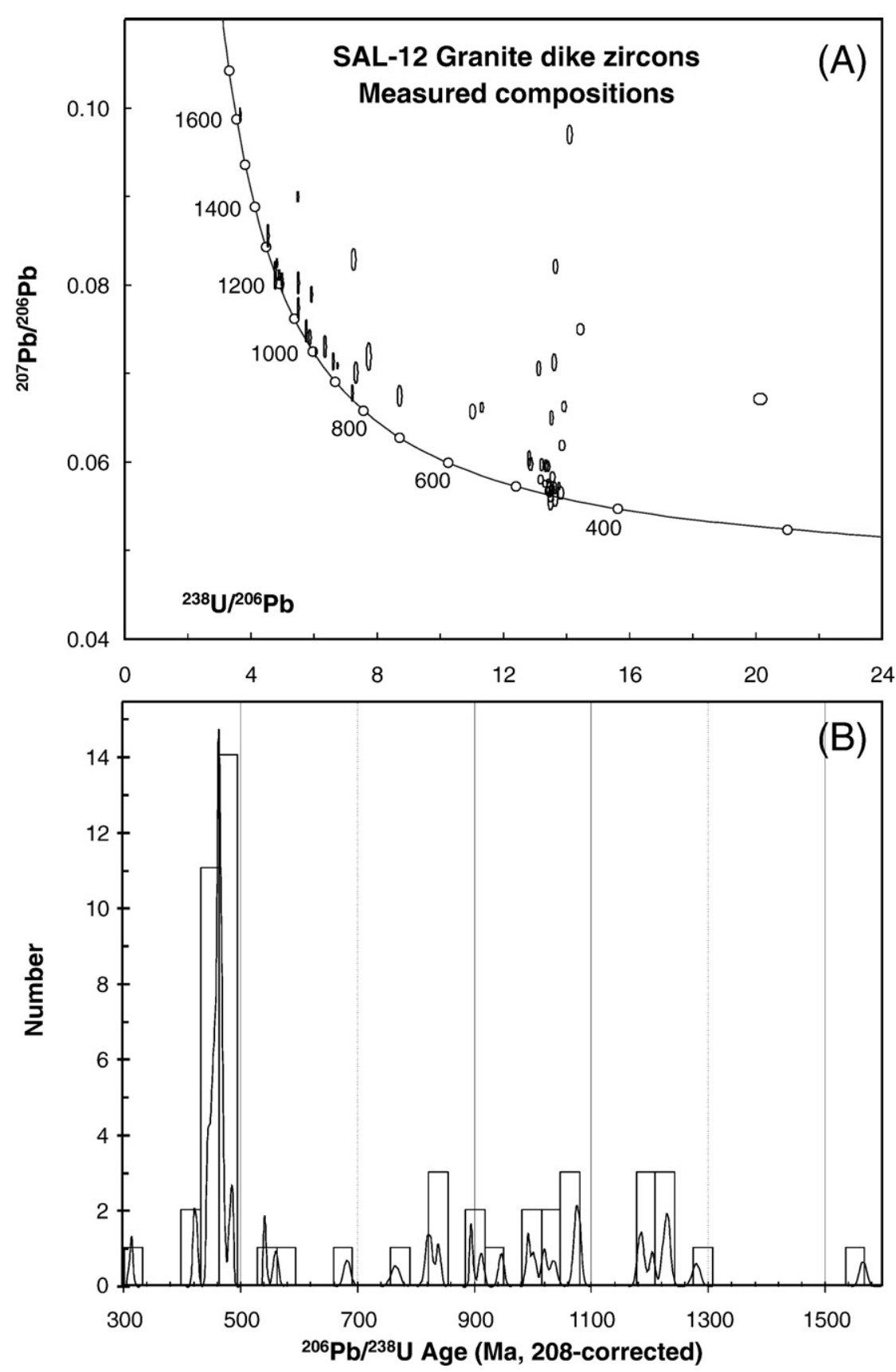

Fig. 7. U-Pb LA-ICPMS zircon analyses from granitic dike cutting the Amate Unit (sample SAL-12) plotted on (A) a Terra-Wasserburg diagram, and (B) a histogram.

The Salada Unit, in addition to these latter populations also contains $\sim 352 \mathrm{Ma}$ detrital zircons. Such ages (353-346 Ma) have been recorded in the zircons from eclogites of the Piaxtla Suite in the central part of the Acatlán Complex (Middleton et al., 2007; Elías-Herrera et al., 2007), where they have been related to exhumation of subduction-related rocks. Thus a local source is likely, however by this time, Pangea had amalgamated and thus more distal sources are also possible.

\subsection{Terminology}

The absence of eclogite facies metamorphism in the Xayacatlán area precludes inclusion of the units in this area in the Piaxtla Suite. Furthermore, the term Xayacatlán is so inextricably linked to the Piaxtla Suite (e.g. Vega-Granillo et al., 2007, and references therein) that we introduce the name, Amate Unit, for the sedimentary rocks previously included in the Xayacatlán Formation. Also the differences in ages of the Huerta and Salada units, both of which were previously assigned to the Cosoltepec Formation (Ortega-Gutiérrez, 1975), justifies their separation and redefinition. A sample of the Cosoltepec Formation type section yielded a similar detrital zircon age population to the Salada Unit, however, the youngest ${ }^{206} \mathrm{~Pb} /{ }^{238} \mathrm{U}$ age reported from the type Cosoltepec Formation is $\sim 376$ Ma (discordant data: Talavera-Mendoza et al., 2005), slightly older than the $352 \mathrm{Ma}$ age in the Salada Unit. Both the 

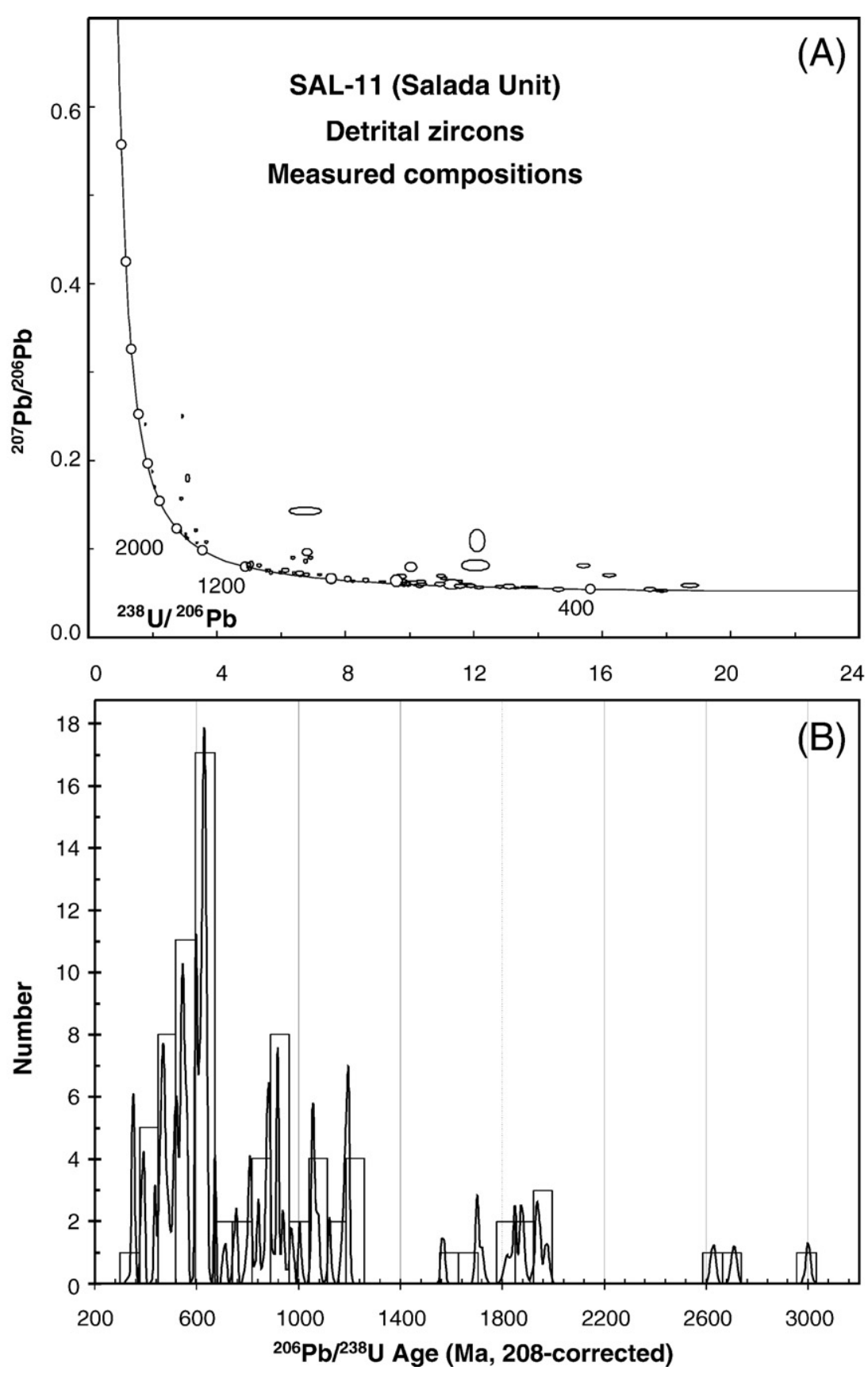

Fig. 8. U-Pb LA-ICPMS zircon analyses from metapsammite of the Salada Unit (sample SAL-11) plotted on (A) a Terra-Wasserburg diagram, and (B) a histogram.

Salada Unit and the type Cosoltepec Formation contain mafic igneous lenses and appear to be continuous along strike from one another (Fig. 1B). However, until mapping and further data confirms their continuity, it is better to maintain the Salada Unit as a distinct unit.

\section{Discussion}

The younger granitoid rocks dated in the Xayacatlán area, which range from $464 \pm 4 \mathrm{Ma}$ to $447 \pm 3 \mathrm{Ma}$ age, are very similar to the $442 \pm 1$ Ma age of a gabbroic dike cutting the Amate Unit (Keppie et al., 2008b). As granitic dikes and pegmatites also cut this mafic dike, it indicates that magmatism in the Xayacatlán area lasted from $\sim 464 \mathrm{Ma}$ to $442 \mathrm{Ma}$. These igneous rocks are bimodal and the mafic dikes are continental rift tholeiites (Keppie et al., 2008b), and appear to form part of a more widespread 480-440 Ma magmatic event in the Acatlán Complex. The Huerta Unit appears to have been deposited during this same time interval, and thus it probably represents a rift setting on the margin of Oaxaquia (Fig. 9). The Oaxacan Complex is inferred to have underlain the Acatlán Complex during the Ordovician (Keppie, 2004), an interpretation that is consistent with the $\sim 1 \mathrm{Ga}$ inherited zircons in the pegmatite cutting the Huerta Unit. The same interpretations probably also apply to the Amate Unit, however, it may be somewhat older. The rifted continental margin setting inferred for all of these 


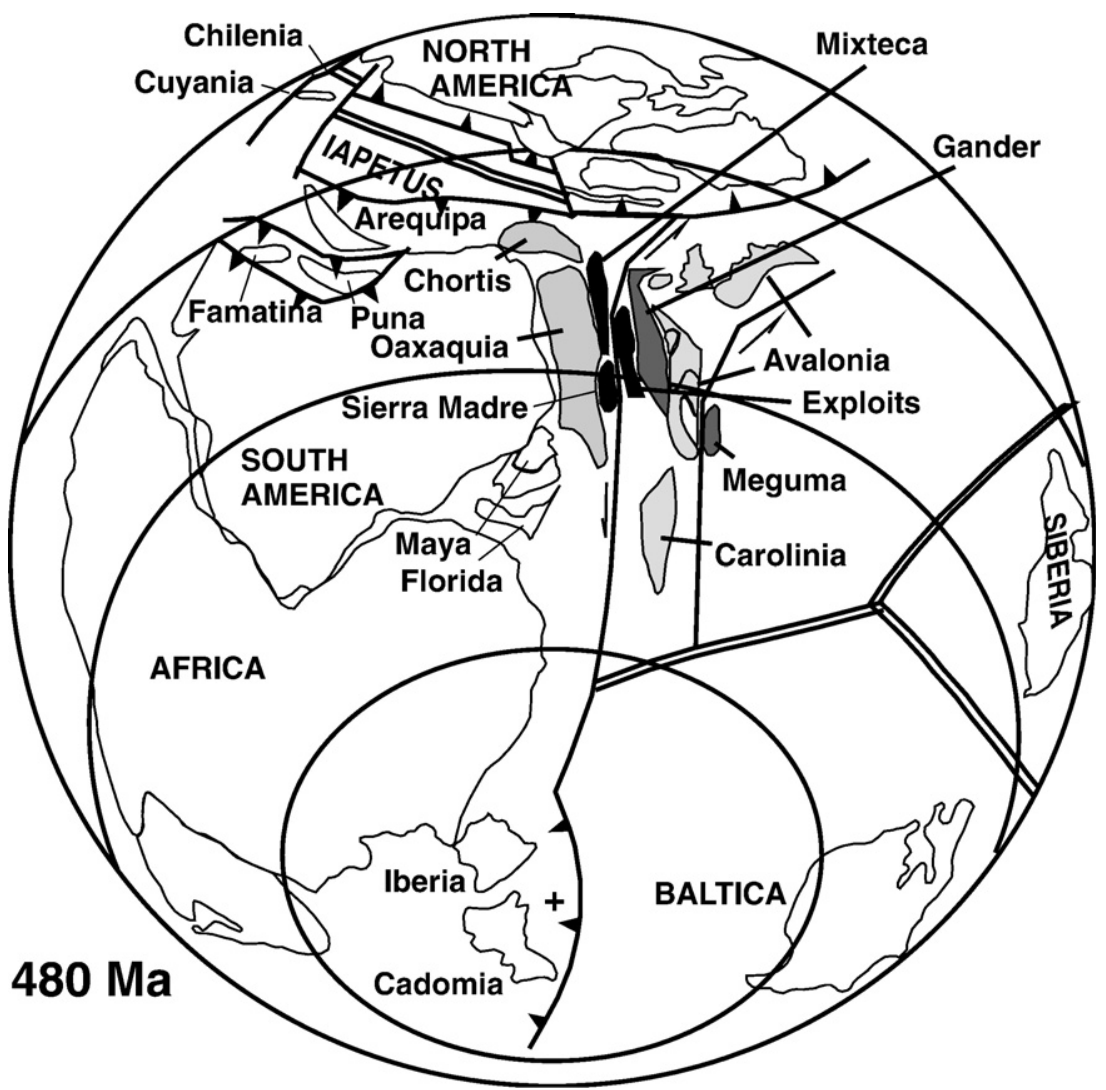

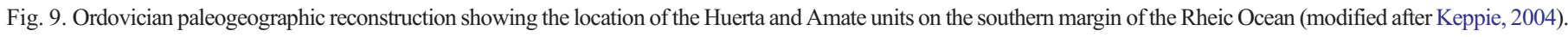

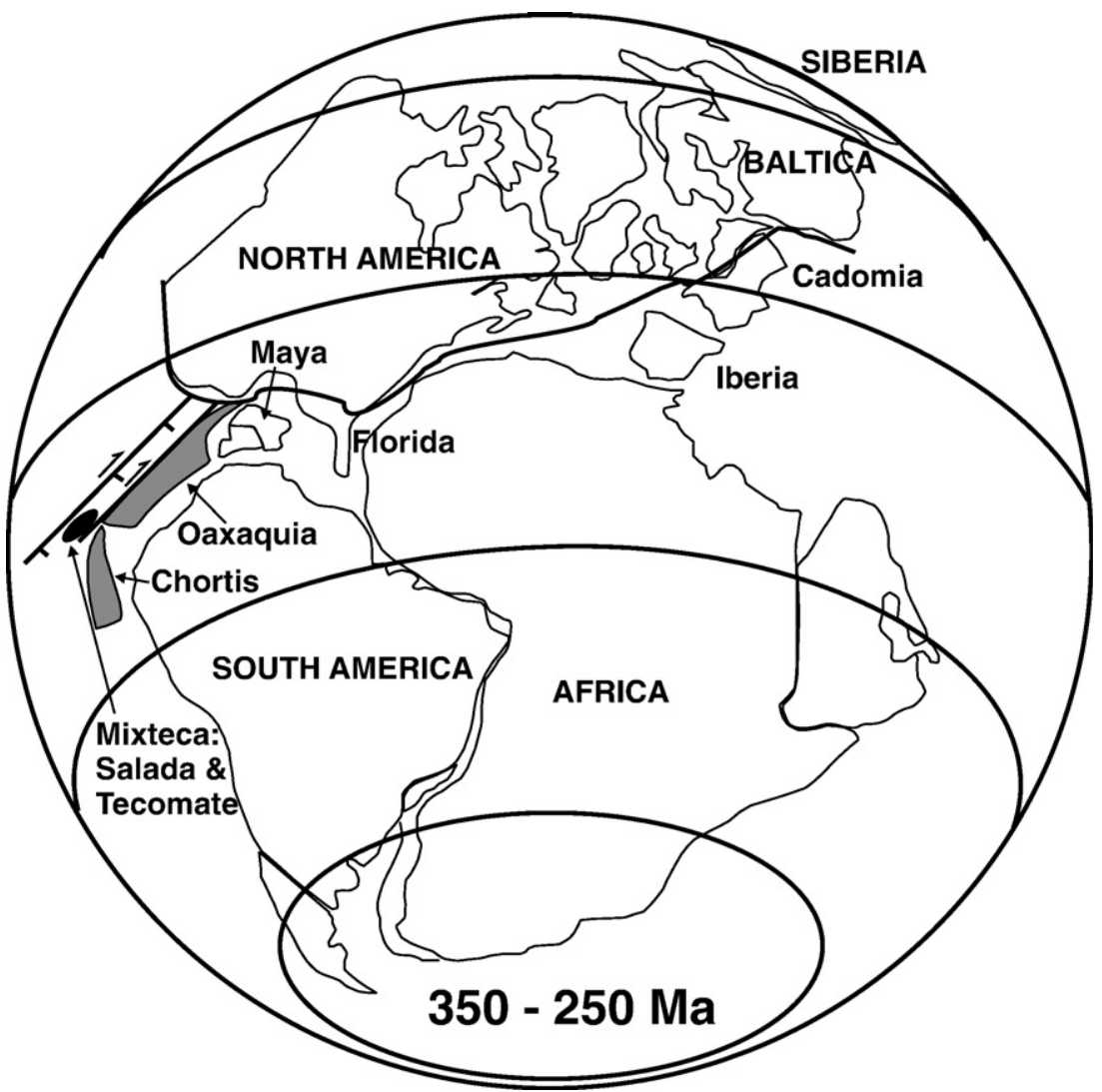

Fig. 10. Carboniferous paleogeographic reconstruction showing the location of the Salada Unit on the western margin of Pangea (modified after Keppie, 2004). 
Ordovician (and possibly older) rocks is more consistent with the deposition along the rift-drift southern margin of the Rheic Ocean (Fig. 9), because the Iapetus Ocean was closing during the Ordovician and it was bordered by active margins. Furthermore, all the detrital zircons in these two units could have been derived from Oaxaquia and Amazonia: the Neoproterozoic zircons are incompatible with the derivation from Laurentia.

The presumed Carboniferous age of the Salada Unit suggests that its deposition was synchronous with the older parts of the latest Devonian-Early Permian Patlanaoaya Group (Vachard et al., 2000), which was synchronous with extensional deformation and easterly dipping subduction (Ramos-Arias et al., 2008). The overlying Permian Tecomate Formation was synchronous with arc magmatism (Torres et al., 1999), including the Totoltepec laccolith. That these rocks formed on the western margin of Pangea (Fig. 10) is indicated by the Midcontinent (USA) faunal affinity of fossils in the Mississippian rocks lying on top of the Oaxacan Complex (Navarro-Santillan et al., 2002).

\section{Acknowledgements}

We would like to acknowledge a Papiit grant (IN103003) and a CONACyT grant (CB-2005-1: 24894) to JDK. CONACyT contributed to studies for MMG. We are grateful for constructive reviews by Drs. Peter A. Cawood and José R. Martínez Catalán.

\section{References}

Campa, M.F., Coney, P.J., 1983. Tectono-stratigraphic terranes and mineral distributions in Mexico. Canadian Journal of Earth Science 20, 1040-1051.

Eggins, S.M., Kinsley, L.P.J., Shelley, J.M.G., 1998. Deposition and element fractionation processes during atmospheric pressure laser sampling for analysis by ICP-MS. Applied Surface Science 127/129, 278-286.

Elías-Herrera, M., Ortega-Gutiérrez, F., 2002. Caltepec fault zone: an Early Permian dextral transpressional boundary between the Proterozoic Oaxacan and Paleozoic Acatlán complexes, southern Mexico, and regional implications. Tectonics 21 (3). doi:10.1029/200TC001278.

Elías-Herrera, M., Macías-Romo, C., Ortega-Gutiérrez, F., Sánchez-Zavala, J.L., Iriondo, A., Ortega-Rivera, A., 2007. Conflicting stratigraphic and geochronologic data from the Acatlán Complex: "Ordovician" granites intrude metamorphic and sedimentary rocks of Devonian-Permian age. Eos Transactions of the American Geophysical Union 88 (23) Joint Assembly Supplement, Abstract T41A-12.

Keppie, J.D., 2004. Terranes of Mexico revisited: a 1.3 billion year odyssey. International Geology Review 46, 765-794.

Keppie, J.D., Ramos, V.S., 1999. Odyssey of terranes in the Iapetus and Rheic Oceans during the Paleozoic. In: Keppie, J.D., Ramos, V.A. (Eds.), Laurentia-Gondwana Connections Before Pangea. Geological Society of America Special Paper, vol. 336, pp. 267-276.

Keppie, J.D., Dostal, J., Cameron, K.L., Solari, L.A., Ortega-Gutiérrez, F., Lopez, R., 2003. Geochronology and geochemistry of Grenvillian igneous suites in the northern Oaxacan Complex, southern México: tectonic implications. Precambrian Research 120, 365-389.

Keppie, J.D., Sandberg, C.A., Miller, B.V., Sánchez-Zavala, J.L., Nance, R.D., Poole, F.G., 2004a. Implications of latest Pennsylvanian to Middle Permian paleontological and $\mathrm{U}-\mathrm{Pb}$ SHRIMP data from the Tecomate Formation to re-dating tectonothermal events in the Acatlán Complex, southern Mexico. International Geology Review 46, 745-753.

Keppie, J.D., Nance, R.D., Powell, J.T., Mumma, S.A., Dostal, J., Fox, D., Muise, J., Ortega- Rivera, A., Miller, B.V., Lee, J.W.K., 2004b. Mid-Jurassic tectonothermal event superposed on a Paleozoic geological record in the Acatlán Complex of southern Mexico: hotspot activity during the breakup of Pangea. Gondwana Research 7, 239-260.

Keppie, J.D., Nance, R.D., Fernández-Suárez, J., Storey, C.D., Jeffries, T.E., Murphy, J.B., 2006. Detrital zircon data from the eastern Mixteca terrane, southern Mexico: evidence for an Ordovician-Mississippian continental rise and a Permo-Triassic clastic wedge adjacent to Oaxaquia. International Geology Review 48, 97-111.

Keppie, J.D., Dostal, J., Murphy, J.B., Nance, R.D., 2008a. Synthesis and tectonic interpretation of the westernmost Paleozoic Variscan orogen in southern Mexico: from rifted Rheic margin to active Pacific margin. Tectonophysics.

Keppie, J.D., Dostal, J., Ramos-Arias, M.A., Morales-Gámez, M., Miller, R.D., Nance, Murphy, J.B., Ortega-Rivera, A., Lee, J.W.K., 2008b. Ordovician rift tholeiites in the Acatlán Complex, southern Mexico: evidence of rifting on the southern margin of the Rheic Ocean. Tectonophysics.

Malone, J.W., Nance, R.D., Keppie, J.D., Dostal, J., 2002. Deformational history of part of the Acatlán Complex: Late Ordovician-Early Silurian and Early Permian orogenesis in southern Mexico. Journal of South American Earth Sciences 15, 511-524.

Middleton, M., Keppie, J.D., Murphy, J.B., Miller, B.V., Nance, R.D., OrtegaRivera, A., Lee, J.K.W., 2007. P-T-t constraints on exumation following subduction in the Rheic Ocean from eclogitic rocks in the Acatlán Complex of southern Mexico. In: Linnemann, U., Nance, R.D., Kraft, P., Zulauf, G. (Eds.), The Evolution of the Rheic Ocean: From Avalonian-Cadomian Active Margin to Alleghenian-Variscan Collision. Geological Society of America Special Paper, vol. 423, pp. 489-509.

Miller, B.V., Dostal, J., Keppie, J.D., Nance, R.D., Ortega-Rivera, A., Lee, J.W.K., 2007. Ordovician calc-alkaline granitoids in the Acatlán Complex, southern Mexico: geochemical and geochronological data and implications for tectonic of the Gondwanan margin of the Rheic Ocean. In: Linnemann, U., Nance, R.D., Zulaf, G., Kraft, P. (Eds.), The Evolution of the Rheic Ocean: From Avalonian-Cadomian Active Margin to Alleghenian-Variscan Collision. Geological Society of America Special Paper, vol. 423, pp. 465-475.

Nance, R.D., Miller, B.V., Keppie, J.D., Murphy, J.B., Dostal, J., 2006. The Acatlán Complex, southern Mexico: record of Pangea assembly to breakup. Geology 34, 857-860.

Nance, R.D., Miller, B.V., Keppie, J.D., Murphy, J.B., Dostal, J., 2007. Vestige of the Rheic Ocean in North America: the Acatlán Complex of southern México. In: Linnemann, U., Nance, R.D., Zulaf, G., Kraft, P. (Eds.), The Evolution of the Rheic Ocean: From Avalonian-Cadomian Active Margin to Alleghenian-Variscan Collision. Geological Society of America Special Paper, vol. 423, pp. 437-452.

Navarro-Santillan, D., Sour-Tovar, F., Centeno-Garcia, E., 2002. Lower Mississippian (Osagean) brachiopods from the Santiago Formation, Oaxaca, Mexico: stratigraphic and tectonic implications. Journal of South American Earth Sciences 15, 327-336.

Ortega-Gutiérrez, F., 1975. The pre-Mesozoic geology of the Acatlán area, south Mexico: Ph.D. thesis, Leeds University, U.K.166 pp.

Ortega-Gutiérrez, F., Elias-Herrera, M., Reyes-Salas, M., Macias-Romo, C., López, R., 1999. Late Ordovician-Early Silurian continental collision orogeny in southern Mexico and its bearing on Gondwana-Laurentia connections. Geology 27, 719-722.

Ortega-Obregón, C., Keppie, J.D., Solari, L.A., Ortega-Gutiérrez, F., Dostal, J., López, R., Ortega-Rivera, A., Lee, J.W.K., 2003. Geochronology and geochemistry of the $\sim 917 \mathrm{Ma}$, calc-alkaline Etla granitoid pluton (Oaxaca, southern México): evidence of post-Grenvillian subduction along the northern margin of Amazonia. International Geology Review 45, 596-610.

Paces, J.B., Miller, J.D., 1993. Precise U-Pb ages of the Duluth Complex and related mafic intrusions, northeastern Minnesota: geochronological insights to physical, petrogenetic, paleomagnetic, and tectonomagmatic processes associated with the 1.1 Ga Midcontinent Rift System. Journal of Geophysical Research 98, 13997-14013.

Ramírez-Espinosa, J., 2001. Tectono-magmatic evolution of the Paleozoic Acatlán Complex in southern Mexico, and its correlation with the Appalachian system: Ph.D. thesis, University of Arizona, $170 \mathrm{p}$.

Ramos-Arias, M.A., Keppie, J.D., Ortega-Rivera, A., Lee, J.W.K., 2008. Extensional deformation on the western margin of Pangea, Patlanoaya area, Acatlán Complex, southern Mexico. Tectonophysics. 
Sánchez-Zavala, J.L., Ortega-Gutiérrez, F., Elías-Herrera, M., 2000. La orogenia Mixteca del Devónico del complejo Acatlán, sur de México. GEOS Unión Geofísica Mexicana, Boletin Informativo Época II, 20, No. 3, pp. 321-322.

Sánchez-Zavala, J.L., Ortega-Gutiérrez, F., Keppie, J.D., Jenner, G.A., Belousova, E., Maciás-Romo, C., 2004. Ordovician and Mesoproterozoic zircons from the Tecomate Formation and Esperanza granitoids, Acatlán Complex, southern Mexico: local provenance in the Acatlán and Oaxacan complexes. International Geology Review 46, 1005-1021.

Solari, L.A., Keppie, J.D., Ortega-Gutiérrez, F., Cameron, K.L., Lopez, R., Hames, W.E., 2003. $\sim 990$ and $\sim 1,100$ Grenvillian tectonothermal events in the northern Oaxacan Complex, southern Mexico: roots of an orogen. Tectonophysics 365, 257-282.

Talavera-Mendoza, O., Ruíz, J., Gehrels, G.E., Meza-Figueroa, D.M., VegaGranillo, R., Campa-Uranga, M.F., 2005. U-Pb geochronology of the Acatlán Complex and implications for the Paleozoic paleogeography and tectonic evolution of southern Mexico. Earth and Planetary Science Letters $235,682-699$.
Torres, R., Ruíz, J., Patchett, P.J., Grajales-Nishimura, J.M., 1999. Permo-Triassic continental arc in eastern Mexico; tectonic implications for reconstructions of southern North America. In: Bartolini, C., Wilson, J.L., Lawton, T.F. (Eds.), Mesozoic Sedimentary and Tectonic History of North-central Mexico. Geological Society of America Special Paper, vol. 340, pp. 191-196.

Vega-Granillo, R., Talavera-Mendoza, O., Meza-Figueroa, D., Ruiz, J., Gehrels, G.E., López-Martínez, M., de La Cruz-Vargas, J.C., 2007. Pressuretemperature-time evolution of Paleozoic high-pressure rocks of the Acatlán Complex (southern Mexico): implications for the evolution of the Iapetus and Rheic Oceans. Geological Society of America Bulletin 119, 1249-1264.

Vachard, D., Flores de Dios, A., Pantoja, J., Buitrón, B.E., Arellano, J., Grajales, M., 2000. Les fusulines du Mexique, une revue biostratigraphique et paléogéographique. Geobios 33, 655-679.

Yañez, P., Ruiz, J., Patchett, J.P., Ortega-Gutiérrez, F., Gehrels, G.E., 1991. Isotopic studies of the Acatlán Complex, southern Mexico: implications for Paleozoic North American tectonics. Geological Society of America Bulletin 103, 817-828. 